\title{
Mass bounds on a very light neutralino
}

\author{
Herbi K. Dreiner ${ }^{1, \mathrm{a}}$, Sven Heinemeyer ${ }^{2, \mathrm{~b}}$, Olaf Kittel ${ }^{3, \mathrm{c}}$, Ulrich Langenfeld ${ }^{4, \mathrm{~d}}$, Arne M. Weber ${ }^{5, \mathrm{e}}$, Georg Weiglein , $^{6, \mathrm{f}}$ \\ ${ }^{1}$ Bethe Center for Theoretical Physics \& Physikalisches Institut der Universität Bonn, Bonn, Germany \\ ${ }^{2}$ Instituto de Física de Cantabria (CSIC-UC), Santander, Spain \\ ${ }^{3}$ Departamento de Física Teórica y del Cosmos and CAFPE, Universidad de Granada, 18071 Granada, Spain \\ ${ }^{4}$ DESY Zeuthen, Zeuthen, Germany \\ ${ }^{5}$ Max-Planck-Institut für Physik (Werner-Heisenberg-Institut), Föhringer Ring 6, 80805 Munich, Germany ${ }^{\mathrm{g}}$ \\ ${ }^{6}$ IPPP, University of Durham, Durham DH1 3LE, UK
}

Received: 12 February 2009 / Revised: 17 April 2009 / Published online: 27 May 2009

(C) Springer-Verlag / Società Italiana di Fisica 2009

\begin{abstract}
Within the Minimal Supersymmetric Standard Model (MSSM) we systematically investigate the bounds on the mass of the lightest neutralino. We allow for nonuniversal gaugino masses and thus even consider massless neutralinos, while assuming in general that $R$-parity is conserved. Our main focus is on laboratory constraints. We consider collider data, precision observables, and also rare meson decays to very light neutralinos. We then discuss the astrophysical and cosmological implications. We find that a massless neutralino is allowed by all existing experimental data and astrophysical and cosmological observations.
\end{abstract}

\section{Introduction}

\subsection{Motivation}

The LHC is scheduled to start taking data in 2009, experimentally opening the window to the TeV energy scale [1]. One of the main goals is to search for physics beyond the standard model (SM). A promising candidate is weak-scale supersymmetry (SUSY), which among other attractive features stabilises the hierarchy between the weak scale and the Planck scale [2]. Supersymmetry predicts a large number of new particles, which, if kinematically accessible, should be observable at the LHC [3-6].

\footnotetext{
a e-mail: dreiner@th.physik.uni-bonn.de

b e-mail: Sven.Heinemeyer@cern.ch

c e-mail: kittel@th.physik.uni-bonn.de

d e-mail: Ulrich.Langenfeld@desy.de

e e-mail: arne@mppmu.mpg.de

f e-mail: Georg.Weiglein@durham.ac.uk

gFormer address.
}

The lightest supersymmetric particle, the LSP, plays a special role in the search for supersymmetry at colliders. If weak-scale supersymmetry is realised in nature, the production rate of the heavier squarks and gluinos will be dominant, due to their strong interactions. But these heavier particles rapidly cascade decay to the LSP in the detector, typically with no detached vertex. Since this occurs in nearly all supersymmetric events, the nature of the LSP and its behaviour are decisive for all supersymmetric signatures at the LHC. Several LSP candidates have been discussed in the literature, e.g. the lightest neutralino, the gluino [7, 8], the lightest stau [9-11], and the lightest sneutrino [12, 13]. In the case of conserved proton hexality, $\mathrm{P}_{6}[14,15]$ (or conserved $R$-parity ${ }^{1}$ [16]) the LSP (and the proton) is stable. A stable light gluino LSP has been excluded by the LEP data [17-19]. A stable (left-handed) sneutrino LSP is also experimentally excluded [20]. Furthermore, cosmologically, a stable LSP must be electrically and colour neutral [21]. This leaves as the most widely studied candidate the lightest neutralino: $\tilde{\chi}_{1}^{0}$, which is a very promising dark matter candidate [21-23]. Further possibilities beyond the MSSM are the gravitino [24-26] or the axino [27-29], which we do not discuss here. We mention as an aside that in the case of ensuring proton stability via baryontriality [30-32] the LSP decays, and in principle any supersymmetric particle can be the LSP. When embedding such models in minimal supergravity the stau, the right-handed smuon, the right-handed selectron, the sneutrino, the lightest sbottom or stop, as well as the right-handed sstrange or sdown have been found to be consistent with experiments [33-36].

In this paper, we focus on a particular aspect of the neutralino LSP, $\tilde{\chi}_{1}^{0}$, namely 'How light can it be?' Our main

\footnotetext{
${ }^{1}$ This is equivalent for the renormalisable superpotential.
} 
interest here is in laboratory constraints, i.e. 'What can one learn about the neutralino mass from collider or fixedtarget experiments?' Thus we initially put aside all aspects of the neutralino as a potential dark matter candidate. We shall discuss the cosmological implications of a very light neutralino towards the end of this paper. One should keep in mind that potential cosmological constraints or requirements can be avoided by adding a small (or large) amount of $R$-parity violation. Potential other dark matter candidates in this case have been discussed in the literature; see for example [37-39]. Unless otherwise indicated, we assume in our analyses that $R$-parity is conserved.

The particle data group (PDG) cites as the laboratory bound on the lightest neutralino mass [40]

$m_{\tilde{\chi}_{1}^{0}}>46 \mathrm{GeV}$

at 95\% C.L., which is based on the searches at LEP (the limit quoted above was obtained in the analysis of the DELPHI collaboration [41]). This has been obtained by taking into account the LEP searches for charginos, which yield lower limits on the $\mathrm{SU}(2)$ gaugino mass parameter, $M_{2}$, and the higgsino mass parameter, $\mu$. Furthermore, this bound assumes an underlying supersymmetric grand unified theory, based on a simple Lie group. In this case, the $\mathrm{U}(1)_{Y}$ gaugino mass parameter $M_{1}$ is related to $M_{2}$ by

$M_{1}=\frac{5}{3} \tan ^{2} \theta_{\mathrm{w}} M_{2} \approx \frac{1}{2} M_{2}$,

so that the experimental bound on $M_{2}$ implies a lower bound on $M_{1}$. Thus the chargino searches yield lower limits on $M_{2}$ and $\mu$ and indirectly on $M_{1}$, which together give rise to a lower bound on the mass of the lightest neutralino, see (1), via the neutralino mass matrix; see (7) below.

It is the purpose of this paper to investigate the consequences of dropping the assumption (2), which invalidates the bound in (1). Such a scenario could occur, for instance, in the case of an underlying string unification with a semi-simple gauged Lie group [42-44]. In this more general scenario, $M_{1}$ and $M_{2}$ are both free parameters. As we shall see, this additional freedom allows for the LEP bound, (1), to be avoided. This raises the question of the corresponding new lower mass bound on the neutralino. Such models have been considered in [45-54], however, while still requiring the neutralino to provide the entire dark matter of the universe. As stated above, our main focus here is on laboratory bounds. We systematically demonstrate that a massless neutralino is consistent with all present laboratory data. We shall consider bounds from direct collider searches, precision electroweak data, and rare meson decays. We then consider the astrophysical (supernova) and cosmological implications of a light neutralino, with a particular focus on a massless neutralino.

\subsection{Outline and connection to previous work}

In early supersymmetric model building, the superpartner of the photon, the photino, $\tilde{\gamma}$, was often considered to be massless or very light (mass below $1 \mathrm{keV}$ ) $[55,56]$. The vanishing mass was guaranteed by a global $R$-symmetry [56], under which the parameters of the supersymmetry transformation, the Grassmann variables $\theta, \bar{\theta}$, transform nontrivially. However, such an $R$-symmetry has several problems. First, it prohibits all gaugino masses, including the gluino mass. As mentioned above, a light gluino has been excluded by LEP data [17-19]. Second, spontaneous breaking of the global $R$-symmetry leads to a problematic axion. The axion and the light gluino can only be evaded by large explicit breaking terms which however render the original symmetry meaningless $[57,58]$. It is possible to gauge a $\mathrm{U}(1) R$-symmetry in $N=1$ local supersymmetry [59]. However in order to cancel the mixed triangle anomalies, we must extend the field content by non-singlet fields under the SM gauge symmetry, or consider a family dependent $\mathrm{U}(1)_{R}[57,58]$; see also [60-62]. In contrast, we shall here consider a very light or massless neutralino, where the small mass comes about through a modest amount of fine-tuning between the parameters $M_{1}$ and $M_{2}$ and consider no further symmetry. This is discussed in detail in Sect. 2. It is then a question of phenomenology, which we will discuss in detail in this paper, to see whether such a light neutralino is consistent with all laboratory, astrophysical and cosmological data. We would also like to point out that models with a light neutralino have recently been obtained in the context of gauge mediated supersymmetry breaking, where heavy messenger masses are provided by the adjoint Higgs field of an underlying $\mathrm{SU}(5)$ grand unified theory [63]. For related work on light neutralinos in other extensions of the MSSM, see, for example, [64, 65].

Following up on the early supersymmetric model building, the phenomenological implications of a stable light photino were mainly discussed in the context of cosmology, namely as a dark matter candidate ${ }^{2}$ [21-23]. Such a photino constitutes hot dark matter. If this would provide the entire dark matter, it is inconsistent with structure formation in the early universe [69-73]. More recently it was found $[45-49,53,54]$ that a light cold dark matter neutralino of mass $\mathcal{O}(5 \mathrm{GeV})$ can be obtained within the MSSM without (2), while still providing dark matter to be con-

\footnotetext{
${ }^{2}$ See also the later work [66-68], where photinos of mass $\mathcal{O}(1 \mathrm{GeV})$ were considered together with a light gluino.
} 
sistent with the WMAP observations [74, 75]. As opposed to the earlier work on a light photino, we consider here a light neutralino; in fact we show that these are predominantly bino, given experimental constraints. Furthermore, we consider significantly lighter, possibly massless, neutralinos. A stable and light neutralino constitutes hot dark matter. For $m_{\tilde{\chi}_{1}^{0}} \ll 25 \mathrm{eV}$ it contributes only a small amount to the energy density of the universe and should thus be consistent with observations. We determine the precise number in Sect. 6.2.1. Another dark matter candidate is then however required [76]. A light photino or neutralino of mass $\mathcal{O}(10 \mathrm{MeV})$ could also be produced in supernova explosions $[77-81] .{ }^{3}$ We reinvestigate these astrophysical and cosmological questions particularly for a massless neutralino in Sect. 6.

As mentioned above, the main focus of this paper is to analyse in detail the issue of laboratory constraints on a light neutralino. We consider collider searches for light neutralinos, the contribution of light neutralinos to precision electroweak observables via radiative corrections and neutralinos in rare meson decays. At colliders, the most promising processes for direct searches are

$e^{+}+e^{-} \rightarrow \tilde{\chi}_{1}^{0} \tilde{\chi}_{1}^{0} \gamma$

$e^{+}+e^{-} \rightarrow e^{+} e^{-} \tilde{\chi}_{1}^{0} \tilde{\chi}_{1}^{0}$.

These were investigated early for a photino $[85,86]$. Three of the present authors $[87,88]$ have recently presented the first complete calculation of the process (3) for a general neutralino, focusing on heavy neutralinos, and analysed the resulting phenomenology at the ILC including beam polarisation. In Sect. 3.1, we reanalyse this for the case of a massless neutralino. We also consider the case of associated production of the lightest and next-to-lightest neutralino $[89,90]$.

A second method to investigate a light neutralino in the laboratory is via its virtual corrections to precision observables. A first point that must be considered when discussing light neutralinos is whether the light mass is stable under radiative corrections. The neutralino also contributes to all electroweak observables via radiative corrections. For early work see for example [91-93], and [94] for a recent review. In most analyses the assumption (2) was typically made; the case of varying the parameter $M_{1}$ independently was not systematically analysed. In general, one would expect that a very light neutralino could give rise to significant supersymmetric corrections to the precision observables. We study this issue in Sect. 4, where we discuss in detail the to-

${ }^{3}$ For a particular application of light but massive photinos see also [82-84]. tal and invisible $Z$ widths, $\Gamma_{Z}$ and $\Gamma_{\mathrm{inv}}$, the $W$ boson mass $M_{W}$, the effective leptonic weak mixing angle $\sin ^{2} \theta_{\text {eff }}$, the anomalous magnetic moment of the muon $(g-2)_{\mu}$ as well as electric dipole moments in the case of complex parameters.

Concerning rare meson decays to neutralinos, the first process studied in the literature was [95-97]

$K \rightarrow \pi \tilde{\chi}_{1}^{0} \tilde{\chi}_{1}^{0}$

For a light neutralino the experimental signature is equivalent to the SM decay $K \rightarrow \pi v \bar{v}$, which has since been observed experimentally [98-101]. The branching ratio still has a large experimental error but is so far consistent with the SM prediction [102-106]. This can thus be used to set bounds on the related supersymmetric parameters. However, typically the decay involves virtual squarks and if these are sufficiently heavy, no bound on the neutralino mass is obtained. For a massless or near massless neutralino, we can consider this instead as a lower bound on the relevant sfermion mass, as in the original study of the anomalous magnetic moment of the muon [107]. In Sect. 5, we present a detailed overview of $K$-meson, pion, $B$-meson, and quarkonium decays [3, 96, 108-115]. A more complete treatment of the bounds resulting from meson decays, including complete (higher order) calculations of the decay rates is deferred to a separate paper [116].

\section{The neutralino framework}

\subsection{Tree-level and higher-order corrections}

We shall work in the minimal supersymmetric SM (MSSM). The part of the Lagrangian which describes the neutralino mixing is given by [5]

$$
\begin{aligned}
\mathcal{L}_{\tilde{\chi}^{0}}= & -\frac{1}{2} \widetilde{\mathcal{B}} \widetilde{\mathcal{B}} M_{1}-\frac{1}{2} \widetilde{\mathcal{W}}^{0} \widetilde{\mathcal{W}}^{0} M_{2}+\mu \widetilde{h}_{1}^{1} \widetilde{h}_{2}^{2} \\
& -\frac{g_{2}}{2} \widetilde{\mathcal{W}}^{0}\left(v_{1} \widetilde{h}_{1}^{1}-v_{2} \widetilde{h}_{2}^{2}\right)+\frac{g_{1}}{2} \widetilde{\mathcal{B}}\left(v_{1} \widetilde{h}_{1}^{1}-v_{2} \widetilde{h}_{2}^{2}\right) \\
\equiv & -\frac{1}{2} \psi_{0}^{T} \mathcal{M}_{\tilde{\chi}_{1}^{0}} \psi_{0},
\end{aligned}
$$

and the fermionic fields are two-component Weyl spinors [117]. Here 
$\mathcal{M}_{\tilde{\chi}^{0}}=\left(\begin{array}{cccc}M_{1} & 0 & -M_{Z} s_{\mathrm{w}} \cos \beta & M_{Z} s_{\mathrm{w}} \sin \beta \\ 0 & M_{2} & M_{Z} c_{\mathrm{w}} \cos \beta & -M_{Z} c_{\mathrm{w}} \sin \beta \\ -M_{Z} s_{\mathrm{w}} \cos \beta & M_{Z} c_{\mathrm{w}} \cos \beta & 0 & -\mu \\ M_{Z} s_{\mathrm{w}} \sin \beta & -M_{Z} c_{\mathrm{w}} \sin \beta & -\mu & 0\end{array}\right)$,

and

$\psi_{0}^{T} \equiv\left(\widetilde{\mathcal{B}}, \widetilde{\mathcal{W}}^{0}, \widetilde{h}_{1}^{1}, \widetilde{h}_{2}^{2}\right)$

$M_{Z}$ is the $Z$ gauge-boson mass and $s_{\mathrm{w}} \equiv \sin \theta_{\mathrm{w}}, c_{\mathrm{w}} \equiv$ $\cos \theta_{\mathrm{w}}=M_{W} / M_{Z}$, where $\theta_{\mathrm{W}}$ is the Weinberg mixing angle. $\mu$ is the higgsino mass parameter and $\tan \beta \equiv v_{2} / v_{1}$ the ratio of the two vacuum expectation values of the Higgs doublets.

The chargino mixing is described by the following matrix [5]:

$\mathcal{L}=-\left(\psi^{-}\right)^{T} X \psi^{+}$

where $\psi^{+} \equiv\left(\tilde{\mathcal{W}}^{+}, \widetilde{h}_{2}^{1}\right)^{T}, \psi^{-} \equiv\left(\tilde{\mathcal{W}}^{-}, \widetilde{h}_{1}^{2}\right)^{T}$,

$X \equiv\left(\begin{array}{cc}M_{2} & \sqrt{2} M_{W} \sin \beta \\ \sqrt{2} M_{W} \cos \beta & \mu\end{array}\right)$.

The mass matrix $X$ is diagonalised by a biunitary transformation:

$\operatorname{diag}\left(m_{\tilde{\chi}_{1}^{ \pm}}, m_{\tilde{\chi}_{2}^{ \pm}}\right)=U^{*} X V^{-1}$

with $U, V$ unitary $2 \times 2$ matrices, see for example [3]. The lower experimental bound on the lightest chargino mass is [40]

$m_{\tilde{\chi}_{1}^{ \pm}}>94 \mathrm{GeV}$

Scanning over the parameter space, taking this bound into account, leads to a lower bound on both $|\mu|$ and $M_{2}$ [64]:

$|\mu|, M_{2} \gtrsim 100 \mathrm{GeV}$.

If the GUT relation between $M_{1}$ and $M_{2},(2)$, is assumed, then this implies

$M_{1} \gtrsim 50 \mathrm{GeV}$

The usually quoted lower bounds on the lightest neutralino mass arise from imposing the experimental bound on the lightest chargino mass, (12), in conjunction with the assumption of the GUT relation between $M_{1}$ and $M_{2}$, (2). The value given in (1) furthermore takes into account results from other searches for supersymmetric particles and constraints from the Higgs sector, see [41] for details.
If instead the theoretical assumption of (2) is dropped and $M_{1}$ and $M_{2}$ are treated as independent free parameters, there is an additional freedom in determining the lightest neutralino mass. In fact we can require a vanishing lightest neutralino mass at tree-level, by setting the determinant of the mass matrix, (7), to zero

$\operatorname{det}\left(\mathcal{M}_{\tilde{\chi}^{0}}\right)=0$

This is equivalent to [118, 119]

$$
\begin{aligned}
\mu & M_{2} M_{Z}^{2} s_{\mathrm{w}}^{2} \sin (2 \beta) \\
& \left.+M_{1}\left(-M_{2} \mu+M_{Z}^{2} c_{\mathrm{w}}^{2} \sin (2 \beta)\right)\right]=0 .
\end{aligned}
$$

The solution $\mu=0$ is excluded by the above chargino bounds. Solving for $M_{1}$ yields

$M_{1}=\frac{M_{2} M_{Z}^{2} \sin (2 \beta) s_{\mathrm{w}}^{2}}{\mu M_{2}-M_{Z}^{2} \sin (2 \beta) c_{\mathrm{w}}^{2}}$.

Accordingly, for fixed values of $\mu, M_{2}$ and $\tan \beta$ one can always find a value of $M_{1}$ such that the lightest neutralino becomes massless. Typically, the second term in the denominator is much smaller than the first, so that the resulting expression for $M_{1}$ is approximately given by (making use of $\sin (2 \beta)=2 \tan \beta /\left(1+\tan ^{2} \beta\right) \approx 2 / \tan \beta$, for $\left.\tan \beta \gtrsim 3\right)$

$$
\begin{aligned}
\mathcal{M}_{1} & \approx \frac{M_{Z}^{2} \sin (2 \beta) s_{\mathrm{w}}^{2}}{\mu} \\
& \approx 2.5 \mathrm{GeV}\left(\frac{10}{\tan \beta}\right)\left(\frac{150 \mathrm{GeV}}{\mu}\right) .
\end{aligned}
$$

This typically implies $M_{1} \ll M_{2}, \mu$. In this parameter region the lightest neutralino $\tilde{\chi}_{1}^{0}$ is predominantly bino, i.e. it couples to hypercharge. The bino admixture is typically above $90 \%$ in the parameter range where the chargino mass bound is satisfied. The masses of the other neutralinos and charginos are of the order of $M_{2}$ and $\mu$, see Fig. 1 .

The results given in (17) and (18) have been obtained at the tree level. Since the chargino/neutralino sector is described by the three independent parameters $M_{1}, M_{2}$ and $\mu$ (in the case of real parameters, and we furthermore assume that $\tan \beta$ is determined via the Higgs sector of the MSSM), only three of the six chargino and neutralino masses are in- 


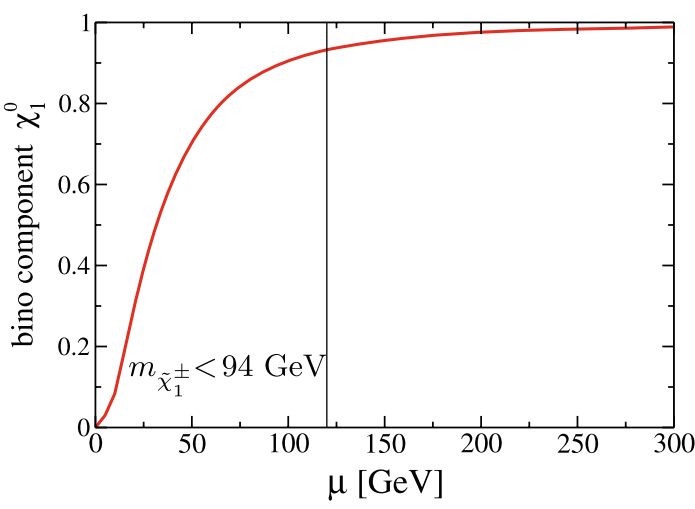

Fig. 1 Bino admixture of $\tilde{\chi}_{1}^{0}$ (left plot) and masses of charginos and neutralinos (right plot) for $M_{2}=200 \mathrm{GeV}, \tan \beta=10$, and $M_{1}$ as given in (17), such that $m_{\tilde{\chi}_{1}^{0}}=0$ [120]. Left of the vertical lines at $\mu \approx 120 \mathrm{GeV}$, the chargino mass is $m_{\tilde{\chi}_{1}^{ \pm}}<94 \mathrm{GeV}$. In the right panel,

dependent. Consequently, the other three masses are predicted. When including radiative corrections, one has to choose a certain renormalisation scheme to define the physical meaning of the parameters. The three independent parameters of the chargino/neutralino mass matrices can be traded for three masses that are specified as input quantities. While in general loop corrections can give rise to a shift between the physical mass and the tree-level mass, the three masses chosen as independent input parameters do not receive higher-order corrections by construction. For the discussion of a very light neutralino it is thus convenient to choose $m_{\tilde{\chi}_{1}^{0}}$ as one of the input parameters in order to avoid that higher-order corrections could drive it away from zero. Such a scheme where the two chargino masses and the lightest neutralino mass have been renormalised on-shell has been applied for the calculation of higher-order corrections in the MSSM chargino/neutralino sector [121, 122]. Accordingly, once the mass of the lightest neutralino has been arranged to be small at tree level, with an appropriate choice of renormalisation scheme it remains small also if higher-order corrections are taken into account. The same also holds in the case of complex parameters, which is discussed in the next subsection.

\subsection{Complex parameters}

The condition for a massless neutralino can also be obtained for a CP-violating neutralino sector. Then the parameters

$M_{1}=\left|M_{1}\right| \mathrm{e}^{i \epsilon \phi_{1}} \quad$ and $\quad \mu=|\mu| \mathrm{e}^{i \epsilon \phi_{\mu}}$

are complex and have CP-violating phases $\phi_{1}$ and $\phi_{\mu}$. We choose the convention where $M_{2}$ is real and positive, absorbing its possible phase by a redefinition of the gaugino fields. In the presence of complex phases, two equations have to be

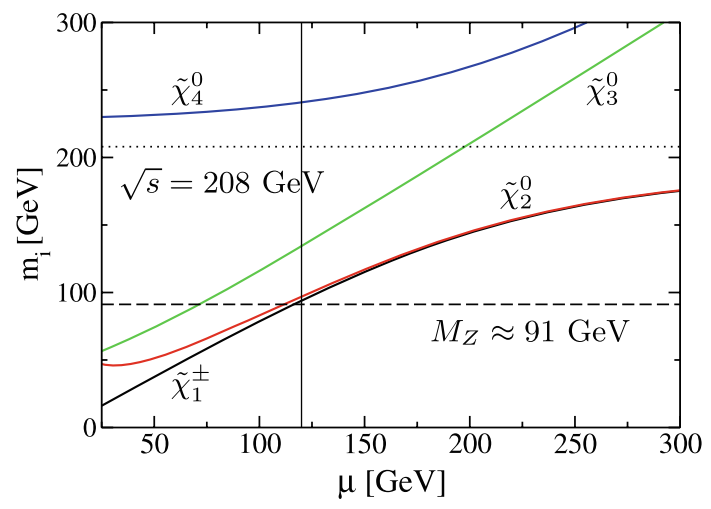

the dotted line indicates the kinematic reach of LEP2 $(\sqrt{s}=208 \mathrm{GeV})$ for $e^{+} e^{-} \rightarrow \tilde{\chi}_{1}^{0} \tilde{\chi}_{i}^{0}$ production $(i=2,3,4)$, and the dashed line indicates the mass of the $Z$ boson, $M_{Z} \approx 91 \mathrm{GeV}$. Note that $\tilde{\chi}_{2}^{0}$ is nearly mass degenerate with $\tilde{\chi}_{1}^{ \pm}$for $\mu>120 \mathrm{GeV}$

separately fulfilled to have a zero mass neutralino,

$\mathfrak{I m}\left\{\operatorname{det}\left(\mathcal{M}_{\tilde{\chi}^{0}}\right)\right\}=0, \quad$ and

$\mathfrak{R e}\left\{\operatorname{det}\left(\mathcal{M}_{\tilde{\chi}^{0}}\right)\right\}=0$.

These conditions are equivalent to

$$
\begin{aligned}
& M_{Z}^{2} c_{\mathrm{w}}^{2} \sin (2 \beta) \sin \phi_{1}-|\mu| M_{2} \sin \left(\phi_{1}+\phi_{\mu}\right)=0, \\
& M_{2} M_{Z}^{2} s_{\mathrm{w}}^{2} \sin (2 \beta)+\left|M_{1}\right|\left[-M_{2} \mu \cos \left(\phi_{1}+\phi_{\mu}\right)\right. \\
& \left.\quad+M_{Z}^{2} c_{\mathrm{w}}^{2} \sin (2 \beta) \cos \phi_{1}\right]=0
\end{aligned}
$$

respectively, and they can be solved for the absolute values of

$$
\begin{aligned}
& |\mu|=\frac{M_{Z}^{2} c_{\mathrm{w}}^{2} \sin (2 \beta) \sin \phi_{1}}{M_{2} \sin \left(\phi_{1}+\phi_{\mu}\right)} \text { and } \\
& \left|M_{1}\right|=-M_{2} \tan ^{2} \theta_{\mathrm{w}} \frac{\sin \left(\phi_{1}+\phi_{\mu}\right)}{\sin \phi_{\mu}} .
\end{aligned}
$$

The equations can also be converted to any other set of two neutralino parameters, for example

$$
\begin{aligned}
& M_{2}=\frac{M_{Z}^{2} c_{\mathrm{w}}^{2} \sin (2 \beta) \sin \phi_{1}}{|\mu| \sin \left(\phi_{1}+\phi_{\mu}\right)} \text { and } \\
& \left|M_{1}\right|=-\frac{M_{Z}^{2} s_{\mathrm{w}}^{2} \sin (2 \beta) \sin \phi_{1}}{|\mu| \sin \phi_{\mu}} .
\end{aligned}
$$

Note that in the presence of non-zero complex phases, the conditions for a massless neutralino cannot always be fulfilled. For example, it follows from (24) that the phases have to fulfil $\sin \phi_{1} / \sin \left(\phi_{1}+\phi_{\mu}\right)>0$ and $\sin \phi_{1} / \sin \phi_{\mu}<0$. In the $\mathrm{CP}$-conserving limit with vanishing phases, we retrieve the condition (17) for $M_{1}$ for a massless neutralino from (22), with $\cos \left(\phi_{1}+\phi_{\mu}\right) \rightarrow 1, \cos \phi_{1} \rightarrow 1$, and (21) is trivially fulfilled. 


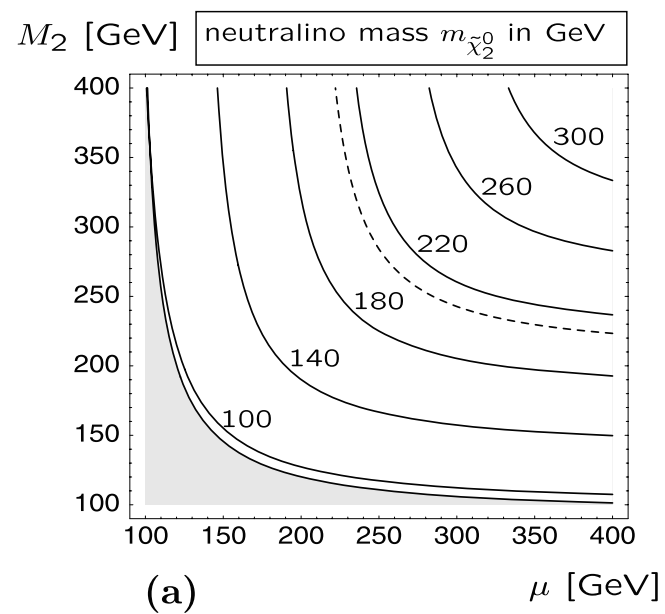

Fig. 2 (a) Contour lines in the $\mu-M_{2}$ plane of the neutralino mass $m_{\tilde{\chi}_{2}^{0}}$. In the grey shaded area the chargino mass is $m_{\tilde{\chi}_{1}^{ \pm}}<94 \mathrm{GeV}$. The dashed line indicates the kinematical limit $m_{\tilde{\chi}_{2}^{0}}=\sqrt{s}=208 \mathrm{GeV}$ at LEP2. Throughout we have chosen $M_{1}$ such that $m_{\tilde{\chi}_{1}^{0}}=0$. The light-

\section{Collider bounds}

In this section, we consider the bounds on a light neutralino from collider searches. We focus our discussion in particular on the direct searches performed by the experimental collaborations at LEP. The bounds from LEP are in general more stringent than the ones from other lepton colliders with lower energies and/or luminosities. We briefly discuss limits from $b$-factories in Sect. 3.2. Concerning bounds from hadron colliders, at the Tevatron the large QCD background limits the sensitivity in the search for direct production of a light or nearly massless neutralino [123]. At the LHC there could be better prospects for detecting effects of a very light neutralino in cascade decays of other (heavy) SUSY particles [124].

\subsection{Neutralino production at LEP}

If we assume a massless neutralino $\tilde{\chi}_{1}^{0}$ by choosing $M_{1}$ as given in (17), the mass of the next-to-lightest neutralino $\tilde{\chi}_{2}^{0}$ is mainly determined by the values of $\mu$ and $M_{2}$. In Fig. 2(a), we show contour lines of the second-lightest neutralino mass. ${ }^{4}$ Qualitatively, the dependence is $m_{\tilde{\chi}_{2}^{0}} \sim M_{2}$ for $M_{2} \ll \mu$, and similarly $m_{\tilde{\chi}_{2}^{0}} \sim \mu$ for $M_{2} \gg \mu$. This can be also observed in Fig. 1, where we show the dependence of the masses on $\mu$. Thus for $\mu$ or $M_{2} \lesssim 200 \mathrm{GeV}$, the associated production of neutralinos, $e^{+} e^{-} \rightarrow \tilde{\chi}_{1}^{0} \tilde{\chi}_{2}^{0}$, cf. Fig. 3,

\footnotetext{
${ }^{4}$ Note that here and for the following scenarios we choose an intermediate value of $\tan \beta=10$ and discuss $\mu>0$ only, since the masses and cross sections change only slightly of the order of $10 \%$ if we take larger values of $\tan \beta$, and/or negative values of $\mu$.
}

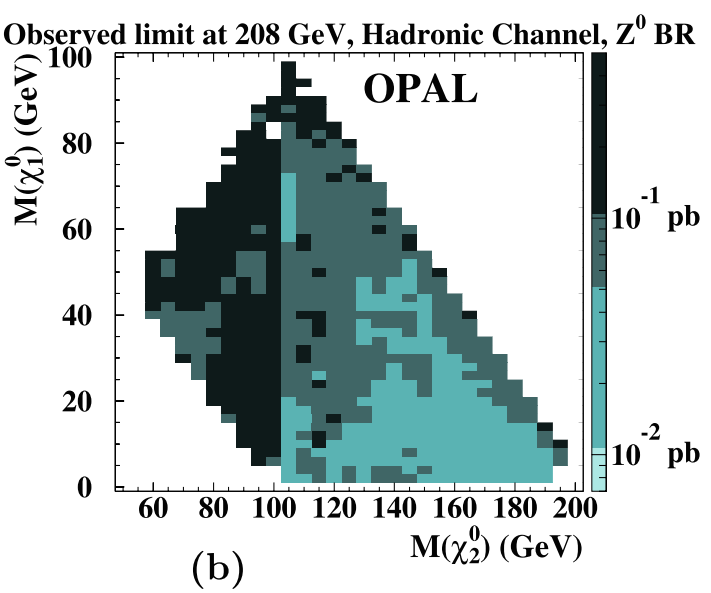

est chargino is nearly mass degenerate with $\tilde{\chi}_{2}^{0}$ for $M_{2} \gtrsim 200 \mathrm{GeV}$ and $\mu \gtrsim 125 \mathrm{GeV}$. (b) $95 \%$ confidence limit on the cross section $\sigma\left(e^{+} e^{-} \rightarrow \tilde{\chi}_{1}^{0} \tilde{\chi}_{2}^{0}\right) \times \mathrm{BR}\left(\tilde{\chi}_{2}^{0} \rightarrow Z \tilde{\chi}_{1}^{0}\right)$ at $\sqrt{s}=208 \mathrm{GeV}$ (taken from [125], Fig. 10)

would be accessible at LEP up to the kinematical limit of $\sqrt{s}=m_{\tilde{\chi}_{2}^{0}}=208 \mathrm{GeV}$, if $m_{\tilde{\chi}_{1}^{0}}=0$.

In order to compare with the results of the LEP searches we make use of the model-independent upper bounds on the topological neutralino production cross section obtained by the OPAL collaboration in the searches at LEP with $\sqrt{s}=$ $208 \mathrm{GeV}$ [125],

$$
\begin{aligned}
& \sigma\left(e^{+} e^{-} \rightarrow \tilde{\chi}_{1}^{0} \tilde{\chi}_{2}^{0}\right) \times \operatorname{BR}\left(\tilde{\chi}_{2}^{0} \rightarrow Z \tilde{\chi}_{1}^{0}\right) \\
& \times \operatorname{BR}(Z \rightarrow q \bar{q}) .
\end{aligned}
$$

In this analysis a stable lightest neutralino is assumed. In Fig. 2(b) the observed limit at 95\% confidence level on $\sigma\left(e^{+} e^{-} \rightarrow \tilde{\chi}_{1}^{0} \tilde{\chi}_{2}^{0}\right) \times \operatorname{BR}\left(\tilde{\chi}_{2}^{0} \rightarrow Z \tilde{\chi}_{1}^{0}\right)$ is shown (taking into account that $\operatorname{BR}(Z \rightarrow q \bar{q}) \approx 70 \%)$ in the $m_{\tilde{\chi}_{1}^{0}}-m_{\tilde{\chi}_{2}^{0}}$ plane [125]. For $m_{\tilde{\chi}_{1}^{0}}=0$ (and $m_{\tilde{\chi}_{2}^{0}} \lesssim 190 \mathrm{Gev}$ ) one can roughly read off the upper limit:

$\sigma\left(e^{+} e^{-} \rightarrow \tilde{\chi}_{1}^{0} \tilde{\chi}_{2}^{0}\right) \times \operatorname{BR}\left(\tilde{\chi}_{2}^{0} \rightarrow Z \tilde{\chi}_{1}^{0}\right)<70 \mathrm{fb}$.

We analyze this bound assuming conservatively that $\operatorname{BR}\left(\tilde{\chi}_{2}^{0} \rightarrow Z \tilde{\chi}_{1}^{0}\right)=1$. In general this branching ratio can be significantly smaller than $100 \%$ since other decay modes like $\tilde{\chi}_{2}^{0} \rightarrow h \tilde{\chi}_{1}^{0}$ and $\tilde{\chi}_{2}^{0} \rightarrow e^{\mp} \tilde{e}^{ \pm}$(see below) can be open. Imposing the bound $\sigma\left(e^{+} e^{-} \rightarrow \tilde{\chi}_{1}^{0} \tilde{\chi}_{2}^{0}\right)<70 \mathrm{fb}$ significantly constrains the parameter space, since the typical neutralino production cross sections are of the order of $100 \mathrm{fb}$ for light neutralino and selectron masses. In Fig. 4(a) we show contour lines of the cross section $\sigma\left(e^{+} e^{-} \rightarrow \tilde{\chi}_{1}^{0} \tilde{\chi}_{2}^{0}\right)$ in the $\mu-M_{2}$ plane for $\tan \beta=10$ and degenerate selectron masses $m_{\tilde{e}_{R}}=m_{\tilde{e}_{L}}=m_{\tilde{e}}=200 \mathrm{GeV}$. We observe that there is a 
Fig. 3 Feynman diagrams for neutralino production $e^{+} e^{-} \rightarrow \tilde{\chi}_{i}^{0} \tilde{\chi}_{j}^{0}$
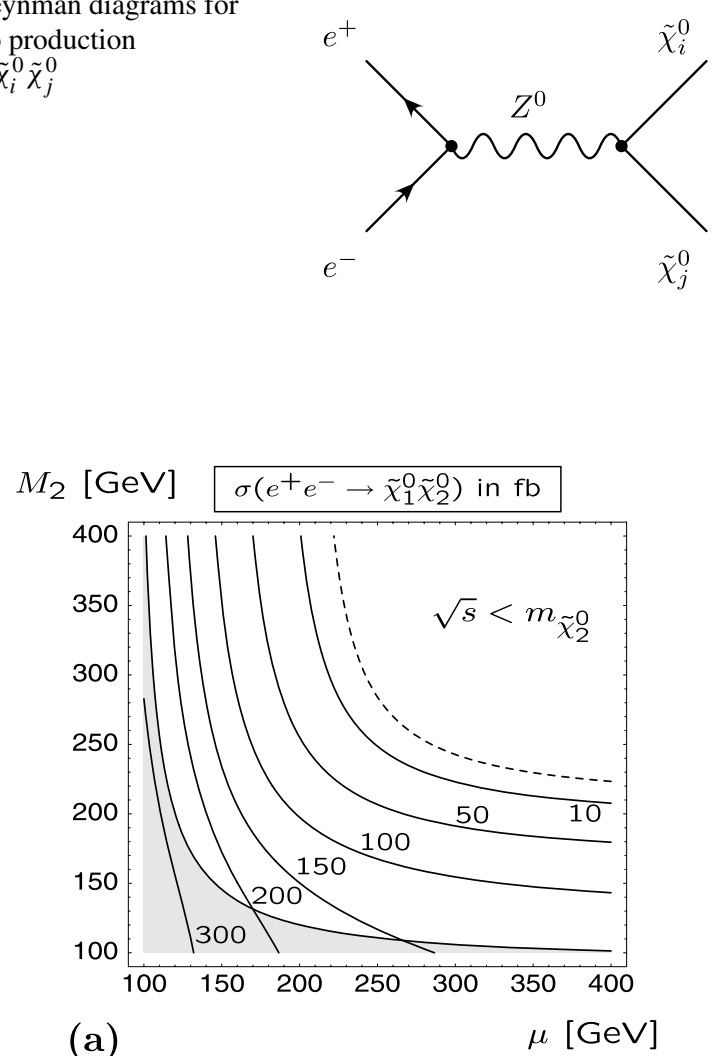

Fig. 4 (a) Contour lines in the $\mu-M_{2}$ plane of the neutralino production cross section $\sigma\left(e^{+} e^{-} \rightarrow \tilde{\chi}_{1}^{0} \tilde{\chi}_{2}^{0}\right)$ with $\tan \beta=10$, and $m_{\tilde{e}_{R}}=m_{\tilde{e}_{L}}=m_{\tilde{e}}=200 \mathrm{GeV}$, at $\sqrt{s}=208 \mathrm{GeV}$. At each point, $M_{1}$ is chosen such that $m_{\tilde{\chi}_{1}^{0}}=0$. (b) Contour lines in the $\mu-M_{2}$ plane of the lower bounds on the selectron mass $m_{\tilde{e}_{R}}=m_{\tilde{e}_{L}}=m_{\tilde{e}}$, such that

large region in the $\mu-M_{2}$ plane where the cross section is higher than $70 \mathrm{fb}$. In order to fulfil the bound, the selectron masses have to be sufficiently heavy. It should be noted that the bino-like $\tilde{\chi}_{1}^{0}$ couples preferably to the $\tilde{e}_{R}$, which is exchanged in the $t$ and $u$ channels, see the Feynman diagrams in Fig. 3. Thus, the bound on the neutralino production cross section can be translated into lower bounds on the selectron mass $m_{\tilde{e}_{R}}=m_{\tilde{e}_{L}}=m_{\tilde{e}}$, for $m_{\tilde{\chi}_{1}^{0}}=0$. In Fig. 4(b), we show contours of the selectron mass, such that the bound $\sigma\left(e^{+} e^{-} \rightarrow \tilde{\chi}_{1}^{0} \tilde{\chi}_{2}^{0}\right)<70 \mathrm{fb}$ is fulfilled. For example, for a fixed selectron mass of $m_{\tilde{e}}=200 \mathrm{GeV}$, the area below the $200 \mathrm{GeV}$ contour in Fig. 4(b) has a cross section in excess of $70 \mathrm{fb}$.

It should be noted that above the dot-dashed contour in Fig. 4(b), the selectron is lighter than the neutralino $\tilde{\chi}_{2}^{0}$. Thus, in that region the two-body decay into a selectron and electron, $\tilde{\chi}_{2}^{0} \rightarrow e^{\mp} \tilde{e}^{ \pm}$, is open, leading to a reduction of $\operatorname{BR}\left(\tilde{\chi}_{2}^{0} \rightarrow Z \tilde{\chi}_{1}^{0}\right)$. The decay of the second-lightest neutralino into a selectron and electron is followed by $\tilde{e}^{ \pm} \rightarrow$ $e^{ \pm} \tilde{\chi}_{1}^{0}$. The reduction of $\operatorname{BR}\left(\tilde{\chi}_{2}^{0} \rightarrow Z \tilde{\chi}_{1}^{0}\right)$ implies a decrease of the lower bound on the selectron mass in this region.
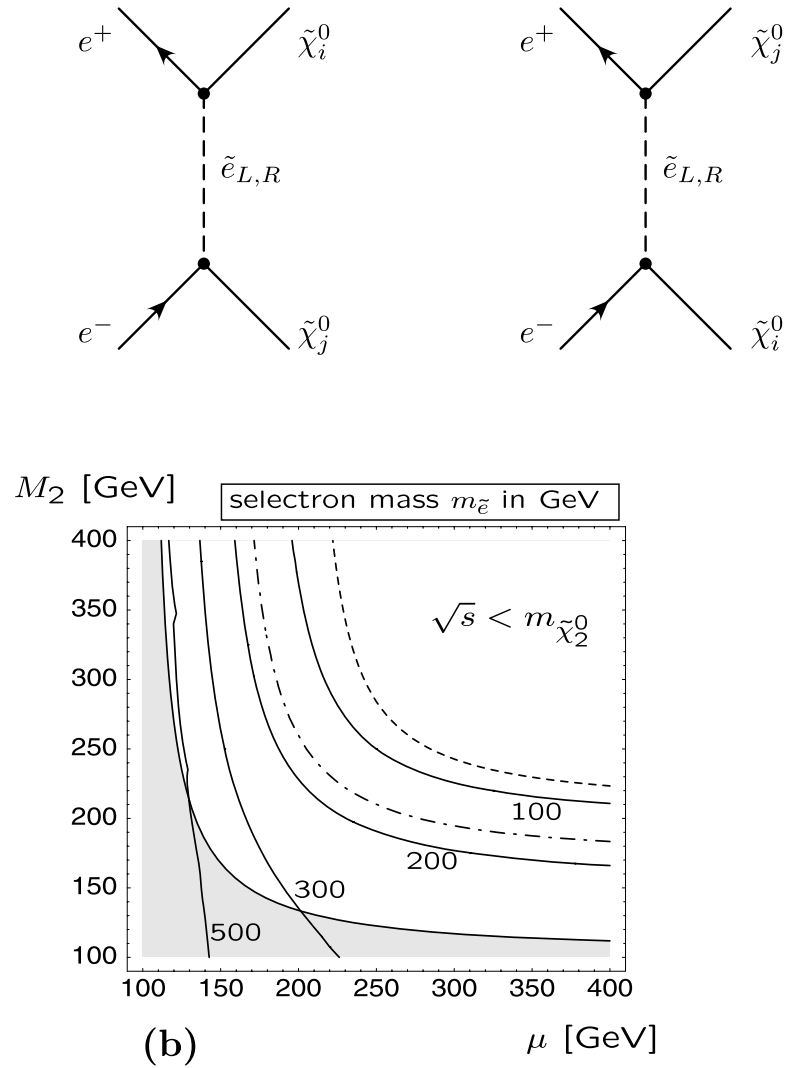

$\sigma\left(e^{+} e^{-} \rightarrow \tilde{\chi}_{1}^{0} \tilde{\chi}_{2}^{0}\right)=70$ fb for $m_{\tilde{\chi}_{1}^{0}}=0$ with $\tan \beta=10$. In (a), (b), the dashed lines indicate the kinematical limit $m_{\tilde{\chi}_{2}^{0}}=\sqrt{s}=208 \mathrm{GeV}$, in the grey shaded areas the chargino mass is $m_{\tilde{\chi}_{1}^{ \pm}}<94 \mathrm{GeV}$. Along the dot-dashed contour in (b) the relation $m_{\tilde{e}}=m_{\tilde{\chi}_{2}^{0}}$ hold

This effect is of minor relevance, however, since the bound on the selectron mass in this region is already close to the absolute lower experimental bound on the selectron mass, $m_{\tilde{e}} \approx 75 \mathrm{GeV}$ [40]. It is clear from our analysis that for a sufficiently heavy selectron mass a massless neutralino could not have been discovered at LEP. Already for $m_{\tilde{e}}=$ $200 \mathrm{GeV}$, we have a significant range of allowed parameter space, as one can see in Fig. 4(b).

Finally, we want to note that the bound on the neutralino production cross section that we have used is indeed rather conservative. Since the decay $\tilde{\chi}_{3}^{0} \rightarrow Z \chi_{1}^{0}$ would also lead to the final state $e^{+} e^{-} \rightarrow q \bar{q}+\mathscr{E}$, the associated production of the next heavier neutralino, $e^{+} e^{-} \rightarrow \tilde{\chi}_{1}^{0} \tilde{\chi}_{3}^{0}$, would increase the signal rate if $m_{\tilde{\chi}_{3}^{0}}<\sqrt{s}$. Since we have approximately $m_{\tilde{\chi}_{3}^{0}} \approx \mu$, see e.g. Fig. 1 , the reaction $e^{+} e^{-} \rightarrow \tilde{\chi}_{1}^{0} \tilde{\chi}_{3}^{0}$ would have been kinematically accessible for $\mu \lesssim 200 \mathrm{GeV}$ at LEP2 with a centre-of-mass energy of $\sqrt{s}=208 \mathrm{GeV}$. Including the additional cross section from $e^{+} e^{-} \rightarrow \tilde{\chi}_{1}^{0} \tilde{\chi}_{3}^{0}$ production in our analysis, we find that for $\mu \lesssim 150 \mathrm{GeV}$ the selectrons must now be heavier than $1 \mathrm{TeV}$. For $\mu \gtrsim$ $200 \mathrm{GeV}$ there is no additional bound. 


\subsection{Radiative neutralino production}

An additional search channel at LEP for a light neutralino would be radiative neutralino production, $e^{+} e^{-} \rightarrow \tilde{\chi}_{1}^{0} \tilde{\chi}_{1}^{0} \gamma$. However due to the large background from radiative neutrino production, $e^{+} e^{-} \rightarrow v \bar{v} \gamma$, we find that the (theoretical) significance ${ }^{5}$ is at best $S \approx 0.1$ for $\mathcal{L}=100 \mathrm{pb}^{-1}$ and $\sqrt{s}=208 \mathrm{GeV}$ [87, 88]. In addition, cuts on the photon energy or angle do not enhance the significance, due to similar kinematic distributions of signal and background. We find a similar situation at $b$-factories, which are operating at the $b$-meson resonances, $\sqrt{s} \approx 10 \mathrm{GeV}$. For example, with $\mathcal{L}=100 \mathrm{fb}^{-1}$ and $m_{\tilde{e}}=200 \mathrm{GeV}$, we find a significance of $S<0.1$, and signal-to-background ratios not larger than $1 \%$. Further, an identification of the signal 'photon plus missing energy' is difficult due to the large photonic background from the abundant hadronic processes at the $b$ factories [126]. Note that at the ILC, however, radiative neutralino production would be measurable, due to the option of polarised beams, which allows one to reduce the background and enhance the signal at the same time [87-90, 127].

\section{Precision observables}

Electroweak observables have in the last decades played a key role in constraining the free parameters of the SM and the MSSM (see e.g. [94] for a recent review in the context of the MSSM). In the following we study the impact of a light or massless neutralino on electroweak precision physics. Among the key observables in the electroweak sector are the mass of the $W$ boson $M_{W}$, the effective leptonic weak mixing angle $\sin ^{2} \theta_{\text {eff }}$ (both discussed in Sect. 4.2), the electric dipole moments of the electron, the neutron, and the mercury atom, and the anomalous magnetic moment of the muon $(g-2)_{\mu}$ (for the latter see Sect. 4.3). The total $Z$ boson decay width $\Gamma_{Z}$ and the invisible $Z$ decay width $\Gamma_{\text {inv }}$ are potentially very sensitive to a massless neutralino, as in such scenarios the additional decay channel $Z \rightarrow \tilde{\chi}_{1}^{0} \tilde{\chi}_{1}^{0}$ is kinematically allowed. The resulting tree-level constraints on a light neutralino were first investigated in [80, 89, 90]. In a first step, in Sect. 4.1 we reanalyse the impact of a massless neutralino on the total width and the invisible width of the $Z$ boson, including full one-loop and leading higher-order corrections.

\subsection{Total $Z$ width $\Gamma_{Z}$ and invisible $Z$ width $\Gamma_{\text {inv }}$}

A light neutralino with mass $m_{\tilde{\chi}_{1}^{0}} \lesssim M_{Z} / 2$ can give contributions to the total and the invisible width of the $Z$ boson,

\footnotetext{
${ }^{5}$ We define the significance $S$ as the number of signal events over the square root of the number of background events.
}

in addition to the decay channels into SM fermions

$$
\begin{aligned}
\Gamma_{Z} & =\underbrace{\Gamma_{Z, \mathrm{SM}}}_{\text {decay into SM fermions }}+\Gamma_{\tilde{\chi}_{1}^{0}}, \\
\Gamma_{\text {inv }} & =\underbrace{\Gamma_{\text {inv, SM }}}_{\text {decay into neutrinos }}+\Gamma_{\tilde{\chi}_{1}^{0}} .
\end{aligned}
$$

Potentially the additional contributions due to $\Gamma_{\tilde{\chi}_{1}^{0}}$ can be large if the neutralino has a considerable non-bino like component, i.e. a sizable coupling to the $Z$ boson. In [128], the processes $Z \rightarrow \tilde{\chi}_{1}^{0} \tilde{\chi}_{1}^{0}$ and $Z \rightarrow f \bar{f}$ have been calculated at $\mathcal{O}(\alpha)$ and supplemented with leading higher-order terms from the SM and the MSSM (see also the discussion below). The corresponding results, which are the currently most accurate MSSM predictions for these quantities, are used in the following to analyse the impact of a massless neutralino on the $Z$ decay width. The experimental values for the total width and the invisible width of the $Z$ boson are [40, 129, 130]

$\Gamma_{Z}^{\exp }=2495.2 \pm 2.3 \mathrm{MeV}$,

$\Gamma_{\text {inv }}^{\exp }=499.0 \pm 1.5 \mathrm{MeV}$.

Below, we label the experimental errors of these two quantities as $\sigma_{\Gamma_{Z}}^{\exp }$ and $\sigma_{\Gamma_{\text {inv }}}^{\exp }$, respectively. In our numerical analysis, we show the results for

$\delta \Gamma_{\mathrm{inv}} \equiv \Gamma_{\mathrm{inv}}-\Gamma_{\mathrm{inv}}^{\exp }$,

$\delta \Gamma_{Z} \equiv \Gamma_{Z}-\Gamma_{Z}^{\exp }$,

i.e. the difference of the MSSM prediction and the experimental result for the invisible $Z$ width, $\delta \Gamma_{\mathrm{inv}}$, and the total $Z$ width, $\delta \Gamma_{Z}$.

In the following, we investigate both $\delta \Gamma_{\text {inv }}$ and $\delta \Gamma_{Z}$ in two representative SUSY parameter regions. As a first scenario, we choose fairly light scalar fermions and set the diagonal soft SUSY-breaking parameter $M_{\text {SUSY }}$ to $250 \mathrm{Gev}$ (in this section we choose this parameter to be equal for all sfermions). In Fig. 5, we show $\delta \Gamma_{\text {inv }}$ in the upper and $\delta \Gamma_{Z}$ in the lower plot, as a function of $M_{2}$ and $\mu . M_{1}$ is fixed ${ }^{6}$ via (17). The remaining SUSY parameters are $\tan \beta=10, A_{\tau}=$ $A_{t}=A_{b}=m_{\tilde{g}}=M_{A}=500 \mathrm{GeV}$. Here $A_{f}(f=t, b, \tau)$ denotes the trilinear couplings of the Higgses to the sfermions, $m_{\tilde{g}}$ is the gluino mass, and $M_{A}$ denotes the mass of the $\mathrm{CP}$-odd Higgs boson. The deviations from the experimental central values as given in (31), (32), are indicated as experimental $n \times \sigma$ contours of the respective observable. In addition, the $95 \%$ C.L. exclusion bounds of $m_{\tilde{\chi}_{1}^{ \pm}}>94 \mathrm{GeV}$ [40] on the chargino mass from direct searches are marked by

\footnotetext{
${ }^{6}$ A negligibly small offset value is added to $M_{1}$ to acquire numerical stability, while scanning the $\mu-M_{2}$ plane.
} 
Fig. 5 The difference of the experimental value and the theory prediction for the invisible $Z$ width, $\delta \Gamma_{\mathrm{inv}}$ (upper plot), and the total $Z$ width, $\delta \Gamma_{Z}$ (lower plot), in the $\mu-M_{2}$ plane, both including the process $Z \rightarrow \tilde{\chi}_{1}^{0} \tilde{\chi}_{1}^{0}$.

Deviations of the theory predictions from the experimental data are indicated as $\delta \Gamma_{\mathrm{inv}} \equiv\left(\Gamma_{\mathrm{inv}}-\Gamma_{\mathrm{inv}}^{\exp }\right)=$ $(10,5,3,2,1) \times \sigma_{\Gamma \text { inv }}^{\text {exp }}$ (upper plot) and $\delta \Gamma_{Z} \equiv$ $\left(\Gamma_{Z}-\Gamma_{Z}^{\exp }\right)=$

$(20,10,3,2,1,0) \times \sigma_{\Gamma 7}^{\exp }$ (lower plot) contours. The SUSY parameters were fixed as $\tan \beta=10, M_{\mathrm{SUSY}}=250 \mathrm{Gev}$, $A_{\tau}=A_{t}=A_{b}=m_{\tilde{g}}=M_{A}=$ $500 \mathrm{Gev}$. For $M_{1}$ we use (17) (see text)
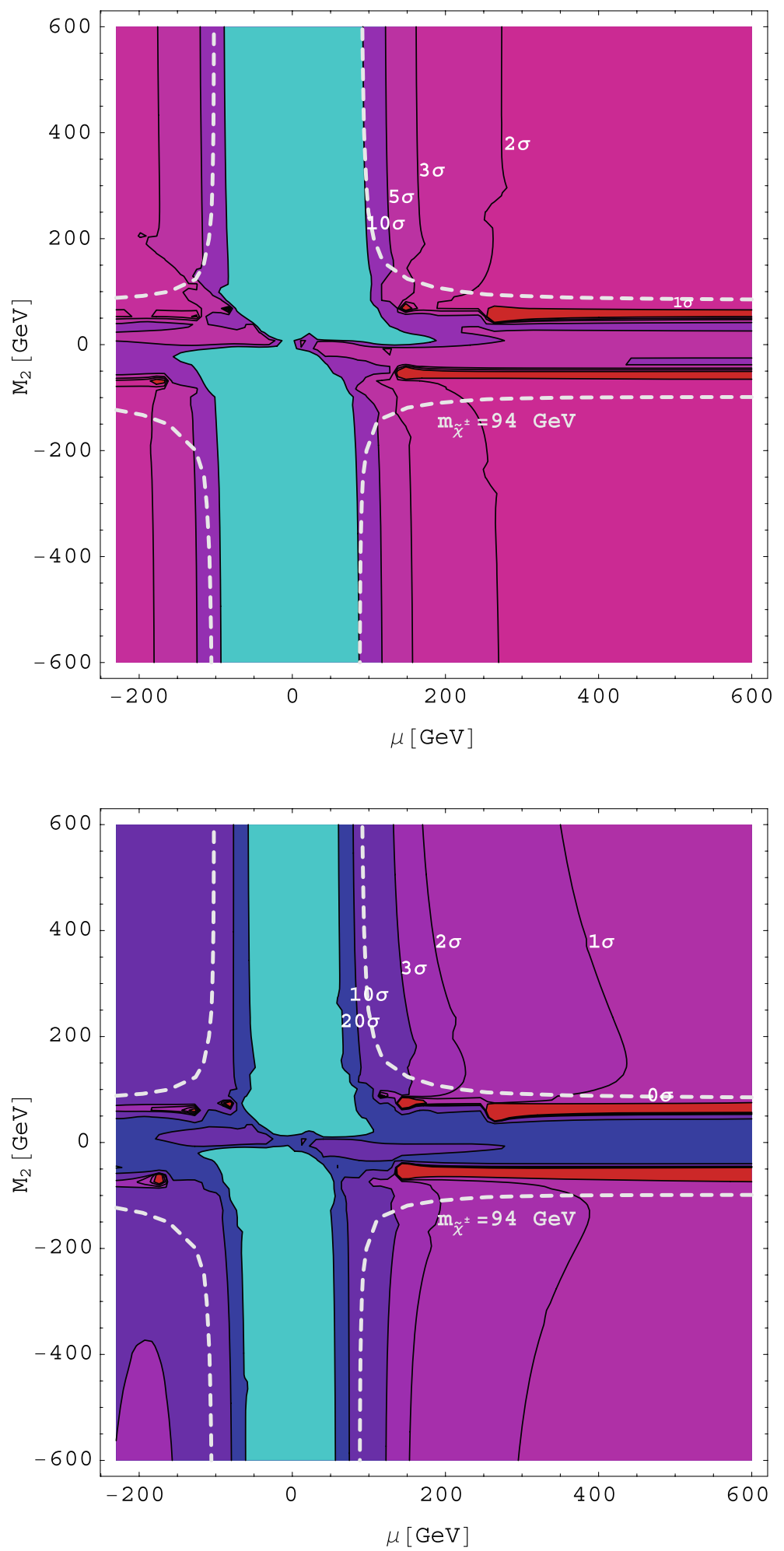

dashed white lines. The allowed regions are towards larger values of $\left|M_{2}\right|$ and $|\mu|$. To the left, the plots stop at around $\mu \approx-230 \mathrm{GeV}$, where the square of the lighter scalar top mass turns negative. Figure 5 clearly displays that for both observables the MSSM prediction can deviate considerably from the experimental values. This is in particular the case for small $|\mu|$ and small $\left|M_{2}\right|$. Nearly all of the parameter space ruled out at the $5 \sigma$ level for $\Gamma_{\mathrm{inv}}$ and at the $3 \sigma$ level for $\Gamma_{Z}$ is, however, already excluded due to direct chargino searches. For the interpretation of these plots it is further- 
more important to keep in mind that the results for $\Gamma_{\mathrm{inv}}$ and $\Gamma_{Z}$ do not only depend on $\mu$ and $M_{2}$, but on all the other SUSY parameters as well. This means in particular that an apparent $1 \sigma$ effect can easily be caused or cancelled out by, for instance, a change induced by $M_{\mathrm{SUSY}}$, the mass scale of the sfermions, which is known to have a strong impact on the decay into SM fermions (see also the discussion in [128]). Furthermore, even in the $\mathrm{SM}, \Gamma_{\mathrm{inv}}$ is predicted to be slightly larger than the experimentally measured value, resulting in $\mathrm{a} \sim 1 \sigma$ deviation.

As a second parameter range, we consider heavier sfermions: $M_{\mathrm{SUSY}}=600 \mathrm{GeV}$. The remaining parameters are given by $\tan \beta=10, A_{\tau}=A_{t}=A_{b}=m_{\tilde{g}}=$ $M_{A}=600 \mathrm{GeV}$. As before, we show in Fig. 6 the results for the invisible $Z$ width $\Gamma_{\text {inv }}$ (upper plot) and the total $Z$ width $\Gamma_{Z}$ (lower plot), as a function of $M_{2} . M_{1}$ is fixed in the same way as before. Here the plot extends beyond $\mu=-600 \mathrm{GeV}$. The theory predictions are again confronted with their experimental values as described above. One finds somewhat better agreement of the $\Gamma_{Z}$ predictions with the experimental data. This is mainly due to the higher mass scale of the scalar fermions, which leads to the prediction of lower $\Gamma_{Z}$ values for a considerable part of the SUSY parameter space (see [128]). Despite the presence of $\Gamma_{\tilde{\chi}_{1}^{0}}$, the overall prediction for $\Gamma_{Z}$ even reaches values below the experimental $1 \sigma$ range, as indicated by the $0 \sigma$ and $-1 \sigma$ contours. $\Gamma_{\text {inv }}$ is in slightly better agreement with the data than before, but a $1 \sigma$ deviation can be observed for the entire allowed $\mu-M_{2}$ plane.

In summary, $\Gamma_{Z}$ and $\Gamma_{\mathrm{inv}}$ cannot exclude a massless neutralino. The parts of the $\mu-M_{2}$ planes that lead to a large deviation from the experimental values are mostly already excluded by direct chargino searches.

\section{2 $W$ boson mass $M_{W}$, effective leptonic mixing angle $\sin ^{2} \theta_{\text {eff }}$ and $\Gamma_{Z}$}

Next we analyse the impact of light neutralinos on $M_{W}$ and $\sin ^{2} \theta_{\text {eff }}$ (and extend the $\Gamma_{Z}$ analysis). The most up-to-date calculations for these quantities were presented in [131] and [128]. These predictions contain the full $\mathcal{O}(\alpha)$ MSSM result, as well as all known MSSM corrections beyond oneloop order, in particular universal contributions entering via $\Delta \rho$ of $\mathcal{O}\left(\alpha \alpha_{s}\right)$ [132, 133] and $\mathcal{O}\left(\alpha_{t, b}^{2}\right)$ [134-137]. Concerning $\sin ^{2} \theta_{\text {eff }}$, in order to reproduce the best available SM results $[138,139]$ in the decoupling limit, also the full electroweak $\mathcal{O}\left(\alpha^{2}\right)$ SM results [140-154], mixed electroweak and QCD SM corrections of $\mathcal{O}\left(\alpha \alpha_{s}\right)[155,156]$ and $\mathcal{O}\left(\alpha \alpha_{s}^{2}\right)$ [157, 158], as well as leading terms beyond two-loop SM [159-164] are accounted for. We show these results as a function of $M_{1}$ in an interval covering the solution of (17), or equivalently given by

$M_{1}=\frac{M_{2} M_{Z}^{2} s_{w}^{2} \sin 2 \beta}{M_{2} \mu-M_{Z}^{2} c_{w}^{2} \sin 2 \beta}+\delta M_{1}$,

with $\delta M_{1}$ ranging roughly from $-100 \mathrm{GeV}$ to $+100 \mathrm{GeV}$. Appropriate choices of $M_{2}$ and $\mu$ allow us to analyse a $\tilde{\chi}_{1}^{0}$ with a substantial bino, zino or higgsino component.

In a first scenario, a bino-like $\tilde{\chi}_{1}^{0}$ is obtained by setting $\tan \beta=10, M_{\mathrm{SUSY}}=250 \mathrm{GeV}, A_{\tau}=A_{t}=A_{b}=\mu=$ $m_{\tilde{g}}=M_{A}=500 \mathrm{GeV}, M_{2}=200 \mathrm{GeV}$ and $M_{1}$ according to (33), with the above mentioned range for $\delta M_{1}$. This results in $m_{\tilde{\chi}_{1}^{0}}$ values between 0 and $\sim 100 \mathrm{GeV}$. What is of interest here is not the absolute value of $M_{W}$ and $\sin ^{2} \theta_{\text {eff }}$ (and $\Gamma_{Z}$ ), which again strongly depends on the remaining SUSY parameters, but the change induced by varying $M_{1}$ and thus also $m_{\tilde{\chi}_{1}^{0}}$. Clearly also these effects are somewhat dependent on e.g. the sfermion mass scale, but the effects due to the neutralino sector are the dominant ones. The results are displayed in Fig. 7, showing the dependence of $M_{W}$ and $\sin ^{2} \theta_{\text {eff }}$ on $M_{1}$ and $m_{\tilde{\chi}_{1}^{0}}$. For complementarity with Sect. 4.1, we have included also the result for $\Gamma_{Z}$. We show the two cases with $\Gamma\left(Z \rightarrow \tilde{\chi}_{1}^{0} \tilde{\chi}_{1}^{0}\right)$ either included or not included into the total $Z$ width. The left column shows the results as a function of $M_{1}$, while the right column as a function of the (corresponding) values of $m_{\tilde{\chi}_{1}^{0}}$. The positive and negative values for $M_{1}$ result each in one branch in the plots on the right. As happens for the chosen set of parameters, $\Gamma_{Z}$ and $M_{W}$ lie fully within the experimental one sigma range, while $\sin ^{2} \theta_{\text {eff }}$ is outside the $1 \sigma$ boundaries everywhere in the plot. As mentioned above, the relative agreement between the MSSM predictions and the experimental results would have been modified if we had chosen different $M_{\text {SUSY }}, A_{t, b}$, or $M_{2}$ (or other SUSY parameters). As can be seen in Fig. 7, none of the observables has a strong dependence on $M_{1}$. The shift induced in $M_{W}$, for example, is $\approx 0.5 \mathrm{Mev}$ for the full $M_{1}$ range. Even with an anticipated ILC precision of $\delta M_{W}^{\mathrm{ILC}}=7 \mathrm{MeV}[165,166]$ this is a marginal effect. The situation is similar for $\sin ^{2} \theta_{\text {eff. }}$. Here one can observe a $\approx 0.4 \times 10^{-5}$ variation with $M_{1}$, which is again marginal even compared with the ILC accuracy of $\delta \sin ^{2} \theta_{\text {eff }}^{\mathrm{ILC}}=1.3 \times 10^{-5}$ [167]. Also the effects induced in $\Gamma_{Z}$, even when including the $Z$-decay to neutralinos, are insignificant compared to the experimental errors, due to the small coupling of the bino-like $\tilde{\chi}_{1}^{0}$ to the $Z$ boson.

As a second representative scenario, we study the case where $\tan \beta=10, M_{\mathrm{SUSY}}=A_{\tau}=A_{t}=A_{b}=M_{A}=m_{\tilde{g}}=$ $600 \mathrm{GeV}, \mu=125 \mathrm{GeV}$, and $M_{2}=200 \mathrm{GeV}$. $M_{1}$ is set according to (33), where $\delta M_{1}=-100 \mathrm{GeV}$ to $+100 \mathrm{GeV}$. For this choice of parameters the positive and negative values for $M_{1}$ result each in one branch in the plots on the right, ranging up to $m_{\tilde{\chi}_{1}^{0}} \lesssim 70-90 \mathrm{GeV}$. The smaller value of $\mu$ here 
Fig. 6 Difference of

experimental value and theory prediction for invisible $Z$ width,

$\delta \Gamma_{\mathrm{inv}}$, (upper plot) and total

$Z$ width, $\delta \Gamma_{Z}$, (lower plot) in

the $\mu-M_{2}$ plane, both including

the process $Z \rightarrow \tilde{\chi}_{1}^{0} \tilde{\chi}_{1}^{0}$.

Deviations of the theory predictions from the

experimental data are indicated as $\delta \Gamma_{\text {inv }} \equiv\left(\Gamma_{\text {inv }}-\Gamma_{\text {inv }}^{\exp }\right)=$ $(10,5,3,2,1.5,1) \times \sigma_{\Gamma}^{\exp }$ (upper plot) and $\delta \Gamma_{\mathrm{inv}} \equiv$ $\left(\Gamma_{Z}-\Gamma_{Z}^{\exp }\right)=$ $(20,10,3,2,1,0,-1) \times \sigma_{\Gamma_{Z}}^{\exp }$ (lower plot) contours. The SUSY parameters are chosen as $\tan \beta=10, M_{\text {SUSY }}=A_{\tau}=$ $A_{t}=A_{b}=m_{\tilde{g}}=M_{A}=$ $600 \mathrm{Gev} . M_{1}$ is defined via (17) (see text)
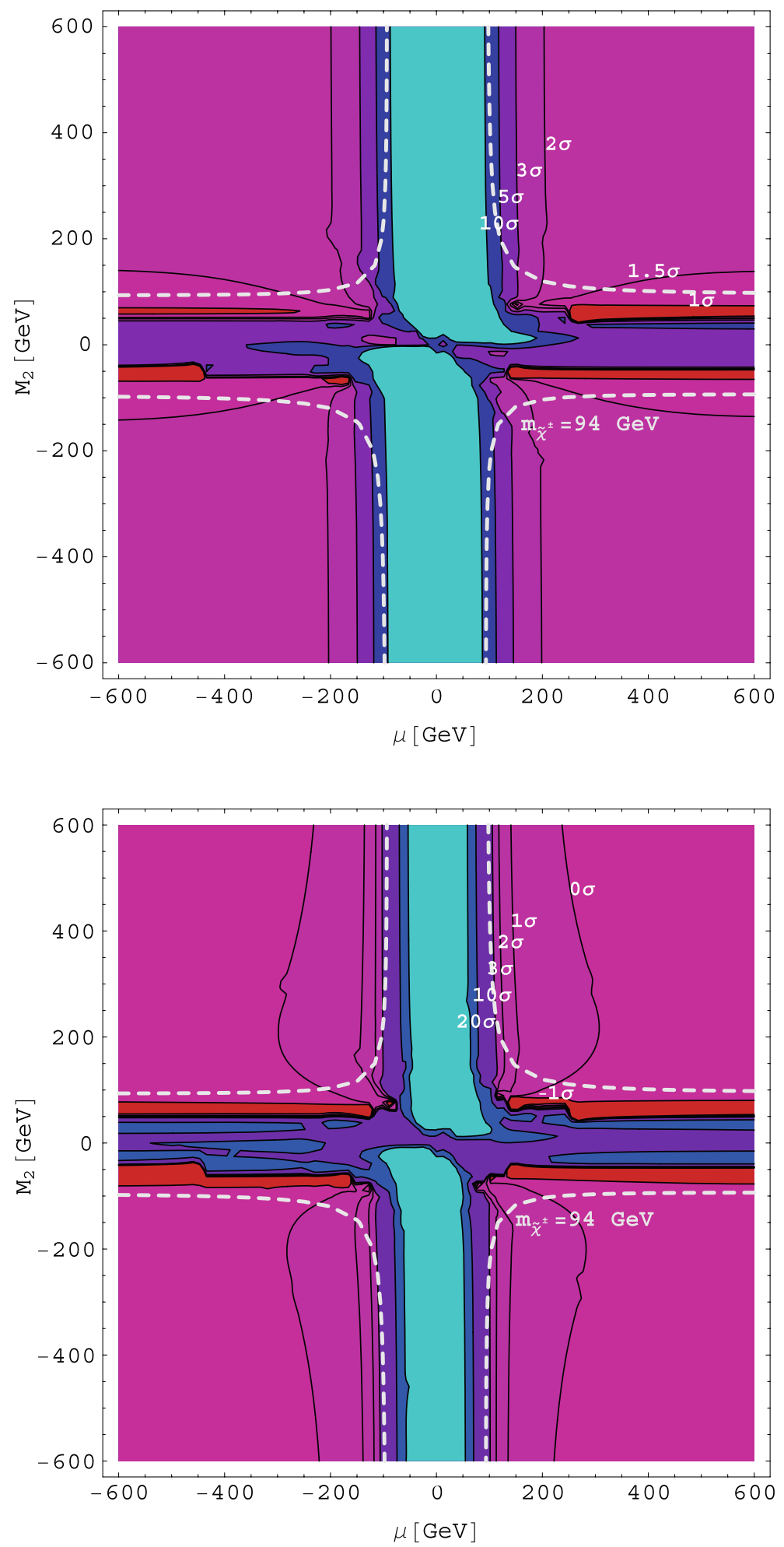

allows for bigger non-bino like $\tilde{\chi}_{1}^{0}$-components. The numerical effects induced in the precision observables are therefore expected to be bigger. This expectation is confirmed by Fig. 8, where as before the left column shows the results as a function of $M_{1}$, while the right column as a function of the (corresponding) values of $m_{\tilde{\chi}_{1}^{0}}$. The variation in $M_{W}$ is $\approx 5 \mathrm{MeV}$, so roughly a factor ten bigger than before. $\sin ^{2} \theta_{\text {eff }}$ shows a variation of $\approx 2 \times 10^{-5}$. Still, even in comparison with the anticipated ILC precisions these are barely observable effects. The largest effects in Fig. 8 can be observed 

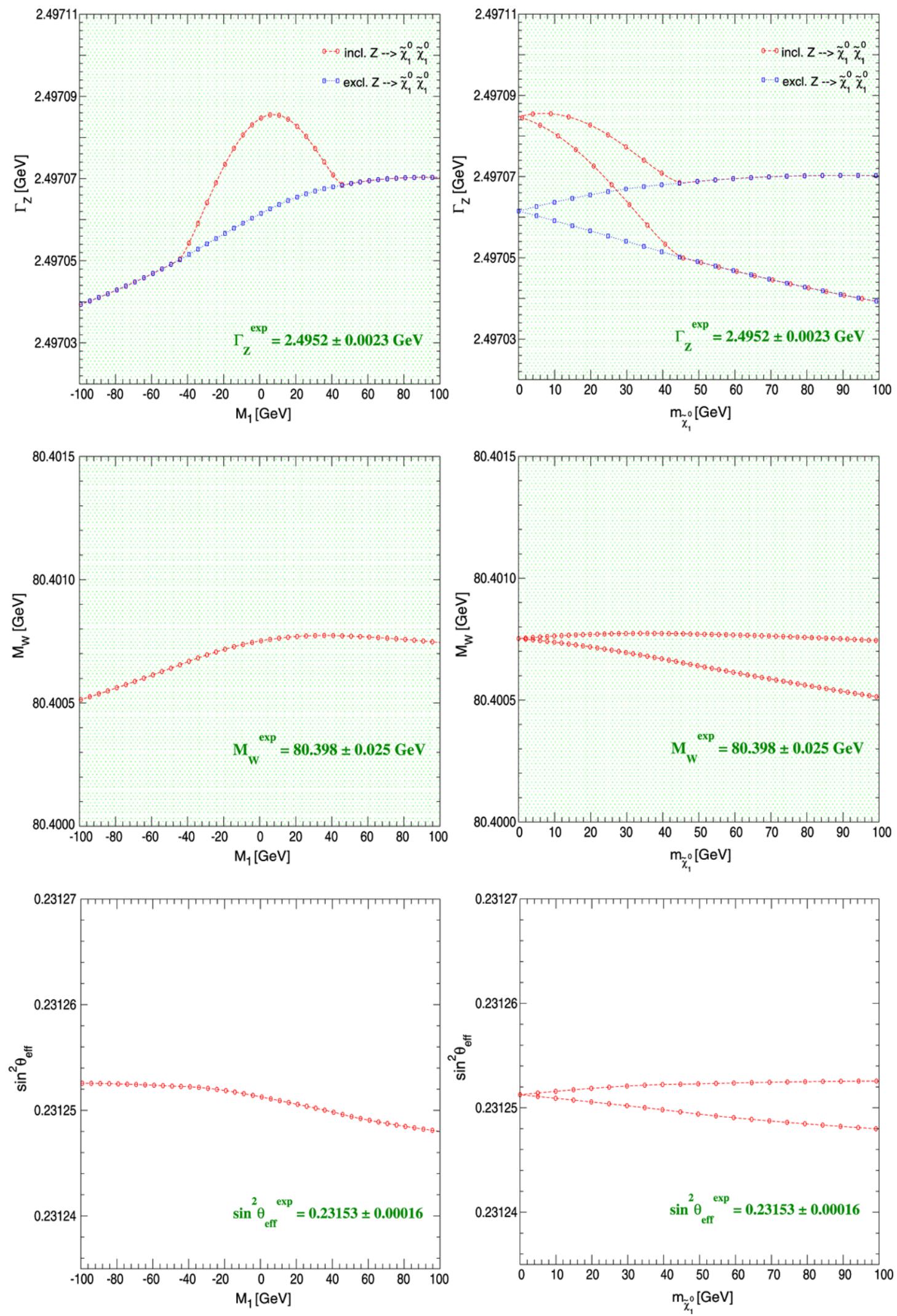

Fig. 7 (Color online) Total $Z$ width $\Gamma_{Z}$ in- and excluding the process $Z \rightarrow \tilde{\chi}_{1}^{0} \tilde{\chi}_{1}^{0}, M_{W}$, and $\sin ^{2} \theta_{\text {eff }}$. The SUSY parameters are chosen thus: $\tan \beta=10, M_{\mathrm{SUSY}}=250 \mathrm{GeV}, A_{\tau}=A_{t}=A_{b}=\mu=m_{\tilde{g}}=M_{A}=$

is varied from -100 to $100 \mathrm{GeV}$, and the two branches in the plots on the right result from negative and positive $M_{1}$. The green shading in the plots for $\Gamma_{Z}$ and $M_{W}$ indicates that the whole area lies within the experimental $1 \sigma$ range 

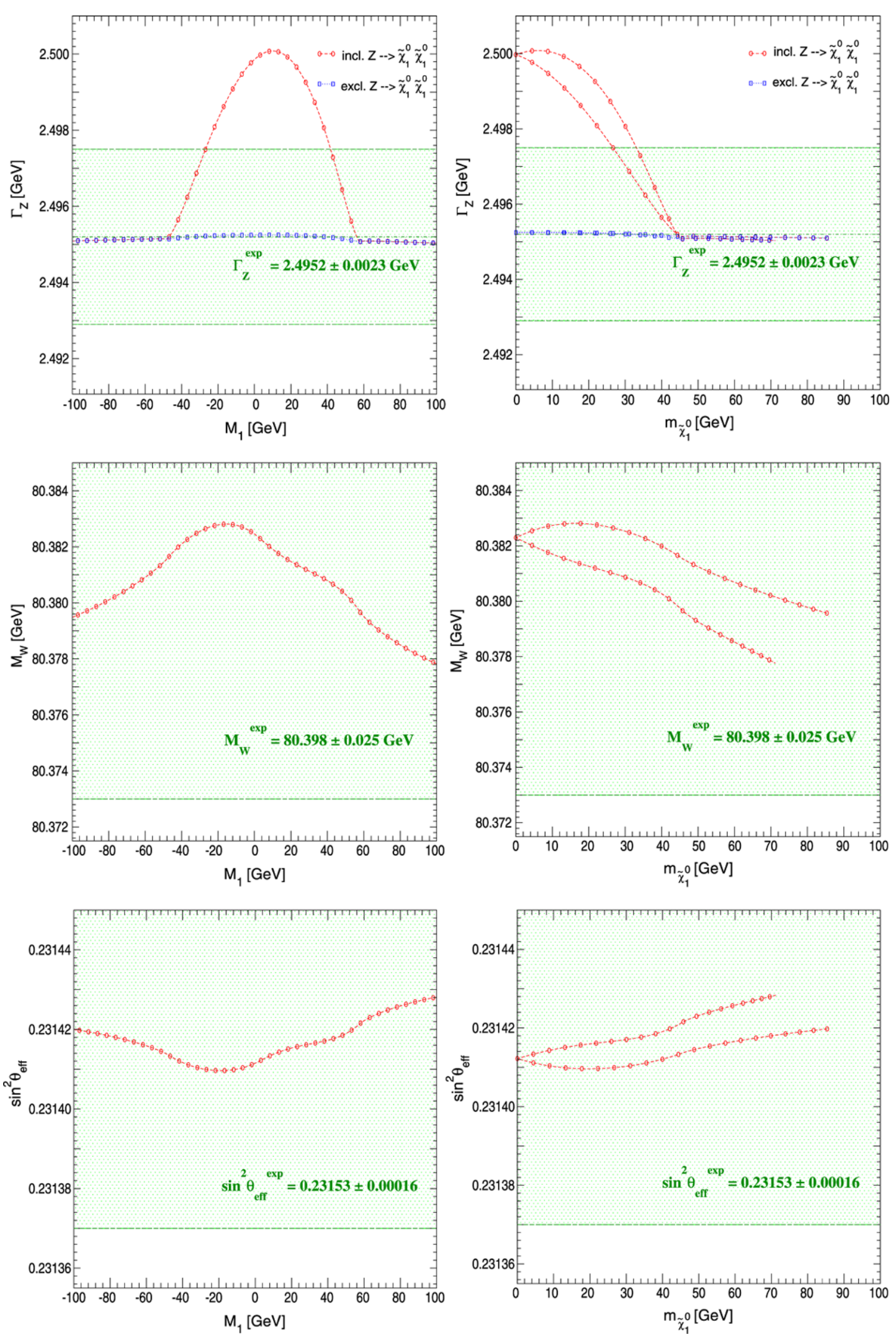

Fig. 8 (Color online) Total $Z$ width $\Gamma_{Z}$ in- and excluding the process $Z \rightarrow \tilde{\chi}_{1}^{0} \tilde{\chi}_{1}^{0}, M_{W}$, and $\sin ^{2} \theta_{\text {eff. The SUSY parameters are chosen to }}$ be $\tan \beta=10, M_{\mathrm{SUSY}}=A_{\tau}=A_{t}=A_{b}=M_{A}=m_{\tilde{g}}=600 \mathrm{GeV}$,

$\delta M_{1}=-100 \mathrm{GeV}$ to $+100 \mathrm{GeV}$, and the two branches in the plots on the right result from negative and positive $M_{1}$. The green shading indicates the experimental $1 \sigma$ range of the three observables 
for the prediction of $\Gamma_{Z}$. The impact of the $Z$-boson decay into a pair of light neutralinos on the total $Z$-boson width is seen to be quite substantial for this choice of SUSY parameters. With today's experimental accuracy, a $2 \sigma$ effect is observable in the presence of a massless neutralino.

To conclude, even the most precise anticipated measurements of $M_{W}$ and $\sin ^{2} \theta_{\text {eff }}$ are not able to constrain the mass of $\tilde{\chi}_{1}^{0}$. The only possible constraints originate from the $Z$ decay width, although even these are not very powerful for most of the parameter space, as they require a rather sizable coupling of $\tilde{\chi}_{1}^{0}$ to the $Z$ boson. Most of the parameter space which fulfils this requirement is already ruled out by direct chargino searches, as discussed in Sect. 4.1. The constraints
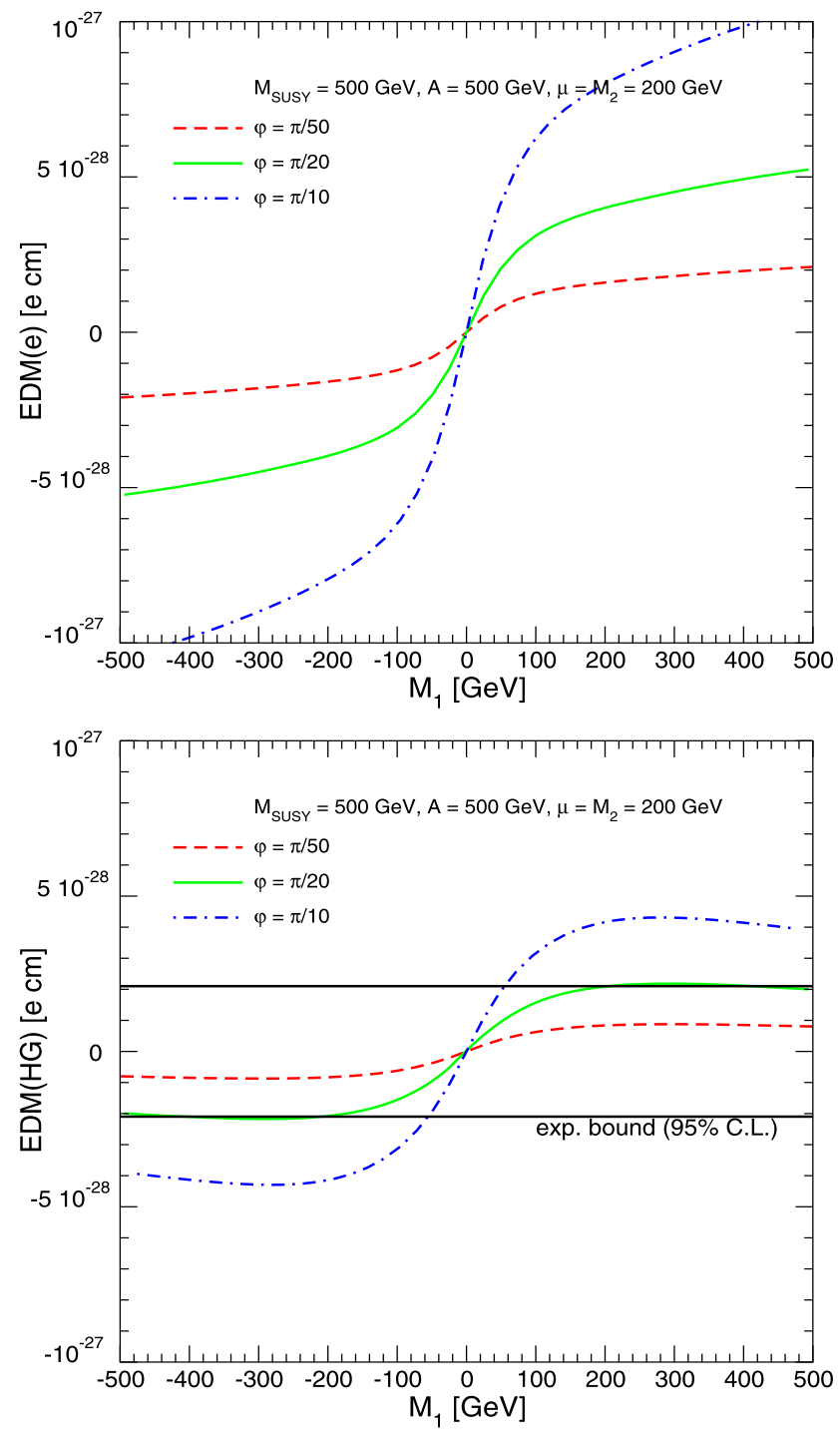

Fig. 9 The EDMs of the electron (upper left plot), the neutron (upper right) and mercury (lower left), as well as the shift in $(g-2)_{\mu}$ normalised to $(g-2)_{\mu}\left(M_{1}=500 \mathrm{GeV}\right)$ (lower right plot), are shown as a function of $M_{1}$ (see text). The other parameters are $M_{H^{ \pm}}=M_{\mathrm{SUSY}}=$ are also easily satisfied by increasing the bino component of the light neutralino. 4.3 Electric dipole moments and anomalous magnetic
moment of the muon

The effect of a small or vanishing mass of the lightest neutralino on electric dipole moments (EDMs) and on the anomalous magnetic moment of the muon, $(g-2)_{\mu}$, is shown in Fig. 9. For the EDMs the one- and two-loop formulas of [168-175] have been used; see [176, 177] for reviews. The SUSY contributions to $(g-2)_{\mu} \equiv 2 a_{\mu}$ are based on the one- and two-loop formulas given in [178-181], see [182]
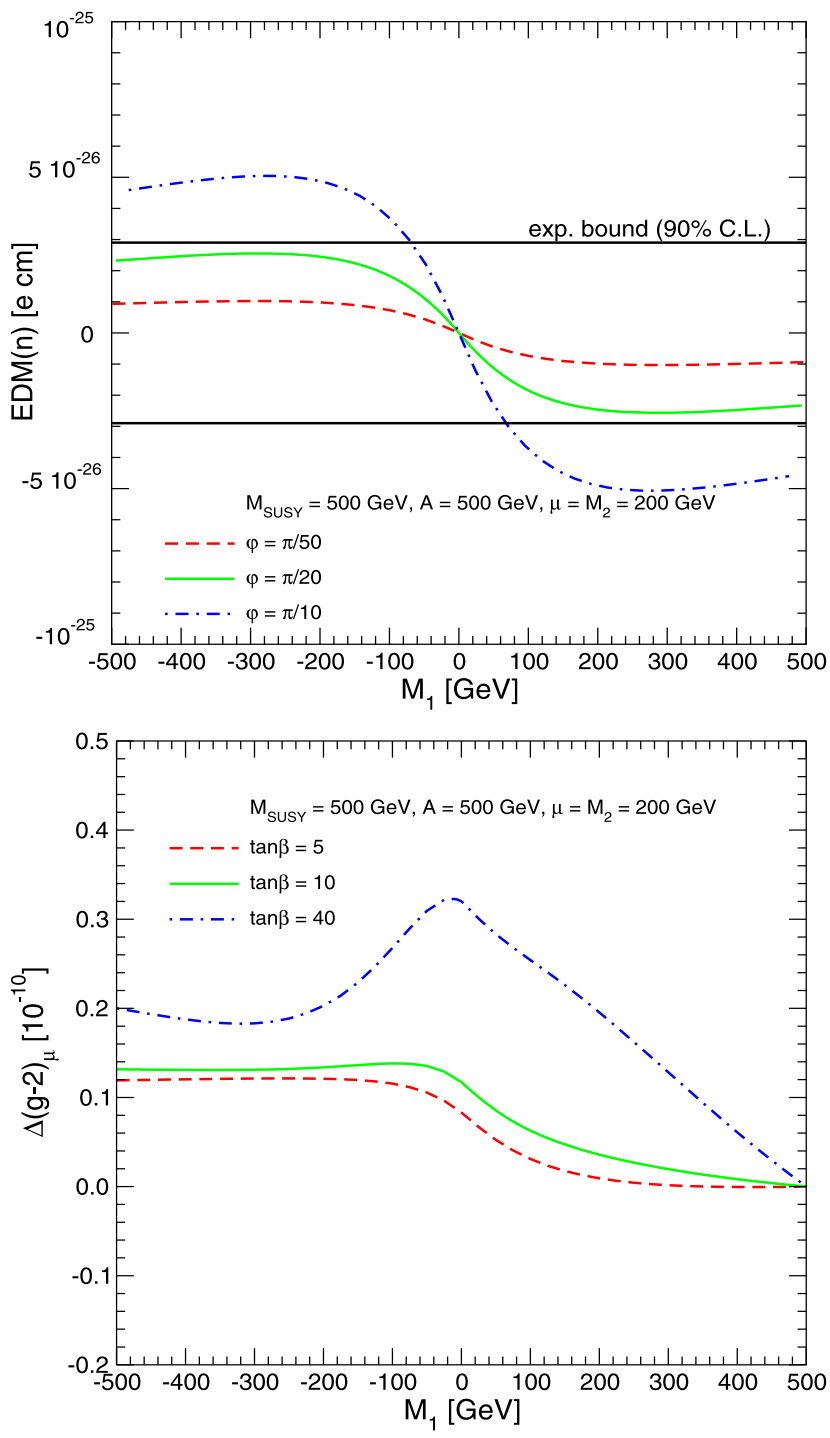

$500 \mathrm{GeV}, A_{f}=500 \mathrm{GeV}, M_{2}=\mu=200 \mathrm{GeV}$. For the EDMs we have fixed $\tan \beta=10$ and set the phase of $M_{1}$ to $\phi_{1}=\pi / 50, \pi / 20, \pi / 10$. For the shift in $(g-2)_{\mu}$ we fixed the phases to be zero, but varied $\tan \beta=5,10,40 . m_{\tilde{\chi}_{1}^{0}}=0$ is reached around $M_{1} \approx 0$ 
Fig. 10 Example diagrams for meson decays into a neutrino pair

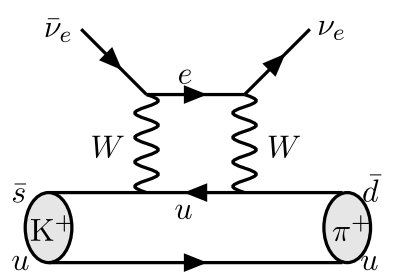

(a) Meson decay into a neutrino pair

(b) $\mathrm{K}^{+}$decay into a neutrino pair and a pion

Table 1 Present bounds for EDMs [192-194]

\begin{tabular}{lll}
\hline System & Limit & Group \\
\hline$e^{-}$ & $1.6 \times 10^{-27}(90 \%$ C.L. $)$ & Berkeley \\
$n$ & $2.9 \times 10^{-26}(90 \%$ C.L. $)$ & ILL \\
${ }^{199} \mathrm{Hg}$ & $2.1 \times 10^{-28}(95 \%$ C.L. $)$ & Seattle \\
\hline
\end{tabular}

for a recent review. These calculations have been performed using the computer code FeynHiggs [183-187].

For the various EDM measurements upper limits exist as given in Table 1. For the anomalous magnetic moment of the muon a "discrepancy" of the experimental result from the SM prediction has been observed [188]

$a_{\mu}^{\exp }-a_{\mu}^{\text {theo }}=(27.5 \pm 8.4) \times 10^{-10}$,

equivalent to a $3.3 \sigma$ effect. $^{7}$ While SUSY contributions could easily explain this "discrepancy", a massless neutralino could in principle lead to a too large contribution to $a_{\mu}$.

The SUSY contribution to the EDMs and to $(g-2)_{\mu}$, shown in Fig. 9, have been evaluated as a function of $M_{1}$ with the other parameters set to $M_{\mathrm{SUSY}}=M_{H^{ \pm}}=500 \mathrm{GeV}$, $A_{f}=500 \mathrm{GeV}, M_{2}=\mu=200 \mathrm{GeV}$. For the EDMs we have fixed $\tan \beta=10$ and set the phase of $M_{1}$ to $\phi_{1}=$ $\pi / 50, \pi / 20, \pi / 10$. For $(g-2)_{\mu}$, we fixed the phases to be zero, but varied $\tan \beta=5,10,40$. According to (17), (18), (23), (24) a massless neutralino is reached around $M_{1} \approx 0$. We show the contribution relative to $(g-2)_{\mu}\left(M_{1}=\right.$ $500 \mathrm{GeV})$, which is $\mathcal{O}\left(10^{-9}\right)$ for this set of parameters.

The SUSY contributions to the EDMS involving the lightest neutralino go to zero for vanishing $m_{\tilde{\chi}_{1}^{0}}$, as can be seen in the upper left, upper right and lower left plot in Fig. 9, where we show the EDM of the electron, the neutron and mercury, respectively. Consequently no lower bound on $m_{\tilde{\chi}_{1}^{0}}$ can be set. For large values of $\phi_{1}$, on the other hand, an upper limit on $M_{1}$ and thus on $m_{\tilde{\chi}_{1}^{0}}$ can be derived as can be seen in the upper right and lower left plot of Fig. 9. The

${ }^{7}$ Three other recent evaluations yield slightly different numbers [189191], but similar discrepancies with the SM prediction. variation of $(g-2)_{\mu}$, shown in the lower right plot of Fig. 9, stays below $\sim 0.5 \times 10^{-10}$ and is thus well below the current uncertainty of $8.4 \times 10^{-10}$ [188]. Therefore, while (for this exemplary set of parameters) the SUSY contributions are of the same size as the deviation between the SM and the experimental result, no experimental limit on $m_{\tilde{\chi}_{1}^{0}}$ can be set.

\section{Rare meson decays}

In the SM, ${ }^{3} S_{1}$ mesons (vector mesons) can decay into a neutrino pair, e.g.

$J / \psi(1 S), \Upsilon(1 S) \rightarrow v_{i} \bar{v}_{i}, \quad i=e, \mu, \tau$,

where $J / \psi$ and $\Upsilon$ denote $c \bar{c}$ and $b \bar{b}$ (ground) states, respectively. Below we also investigate $\phi=s \bar{s}$ and the light mesons $\rho$ and $\omega$, which are superpositions of $u \bar{u}$ and $d \bar{d}$ states. An example the Feynman graph for a meson decay into a neutrino pair at the parton level is shown in Fig. 10(a). If we allow for neutrino masses, which enable a chirality flip, also ${ }^{1} S_{0}$ mesons (pseudoscalar mesons) can decay into a neutrino pair, e.g. $[195,196]$

$\pi^{0} \rightarrow v_{i} \bar{v}_{i}, \quad i=e, \mu, \tau$,

where again Fig. 10(a) represents the tree-level graph. In both cases, the neutrino pair remains unobservable. It is possible to look for the invisible decay of mesons, (35), (36), for example via the decay chain

$$
\begin{aligned}
\psi(2 S) \rightarrow & J / \psi+\pi \pi \\
& \hookrightarrow v_{i} \bar{\nu}_{i}, \quad i=e, \mu, \tau,
\end{aligned}
$$

by tagging on the invariant mass of the di-pion system. To our knowledge, this idea was first proposed in [197], as a test of the SM neutral current, and it has since been widely employed [198-201]. In [202] it was proposed as a method to look for physics beyond the SM, then a light gravitino. It has since been used to look for many different aspects of beyond the SM physics [110-115, 203-205]. 
Fig. 11 Example diagrams for the decay of a $\pi, B_{s}$, and $J / \psi$-meson into a bino pair

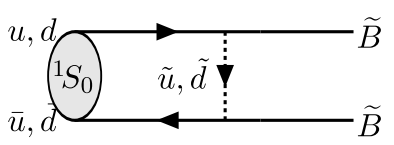

(a) pion decay

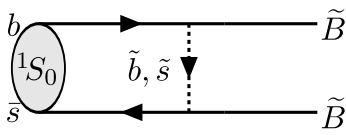

(b) $B_{s}$ decay

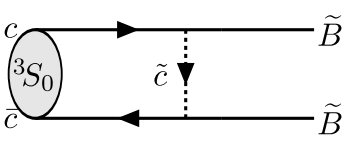

(c) $J / \psi$ decay
Table 2 Comparison of maximum values of various branching ratios of meson decays to light binos. We show the theoretical maximum value and the current experimental bounds on the BR. For the theoretical computation we choose the bino mass to maximise the BR. The upper (middle) part shows the results for the pseudoscalar (vector) mesons, the lower part shows a rare decay. For the pseudoscalar mesons, the squark masses are set to $300 \mathrm{GeV}$. For the vector mesons, see (47)-(51). All data are taken from [40,213] and from the specified literature. "-" indicates that no experimental bound has been derived yet. We have included also for comparison a related experimental result on the invisible decay of the $\Upsilon[113,114]$

\begin{tabular}{llll}
\hline Decay & Maximal BR & Experimental bound on BR & Literature \\
\hline$\pi^{0} \rightarrow \widetilde{B} \widetilde{B}$ & $\mathcal{O}\left(10^{-13}\right)$ & $<2.7 \times 10^{-7}$ & {$[108,109,196,214]$} \\
$\eta \rightarrow \widetilde{B} \widetilde{B}$ & $\mathcal{O}\left(10^{-13}\right)$ & $<6 \times 10^{-4}$ & {$[200]$} \\
$\eta^{\prime} \rightarrow \widetilde{B} \widetilde{B}$ & $\mathcal{O}\left(10^{-14}\right)$ & $<1.4 \times 10^{-3}$ & {$[200]$} \\
$B_{S} \rightarrow \widetilde{B} \widetilde{\mathcal{B}}$ & $\mathcal{O}\left(10^{-8}\right)$ & - & {$[110-112]$} \\
$\phi \rightarrow \widetilde{B} \widetilde{B}$ & $\mathcal{O}\left(10^{-16}\right)$ & - & {$[209]$} \\
$J / \psi \rightarrow \widetilde{B} \widetilde{B}$ & $\mathcal{O}\left(10^{-11}\right)$ & $<7.1 \times 10^{-4}$ & {$[201,209]$} \\
$\Upsilon(1 S) \rightarrow \widetilde{B} \widetilde{B}$ & $\mathcal{O}\left(10^{-10}\right)$ & $<2.5 \times 10^{-3}$ & {$[209,215-217]$} \\
$\Upsilon(1 S) \rightarrow$ inv. & & - & {$[113,114]$} \\
$\rho \rightarrow \widetilde{B} \widetilde{B}$ & $\mathcal{O}\left(10^{-15}\right)$ & - & {$[95,96]$} \\
$\omega \rightarrow \widetilde{B} \widetilde{B}$ & $\mathcal{O}\left(10^{-16}\right)$ & $1.5_{-0.9}^{+1.3} \times 10^{-10}$ & \\
$K^{+} \rightarrow \pi \widetilde{B} \widetilde{B}$ & $\mathcal{O}\left(10^{-15}\right)$ &
\end{tabular}

Mesons can also decay to a lighter meson and a neutrino pair, e.g. $[99,101]$

$K^{+} \rightarrow \pi^{+} \nu_{i} \bar{\nu}_{i}, \quad i=e, \mu, \tau$

A parton level Feynman graph is shown in Fig. 10(b). The rare decay, (38), has a similar signature to the cascade, (37), and has in fact been observed [99, 101]. The partial decay width is consistent with the SM [206, 207], albeit with a still large experimental error. It has also been used to investigate beyond the SM physics [95-97, 208-211].

In the $\mathrm{P}_{6}$ conserving MSSM with a stable, light neutralino, we can replace the neutrino pair in the decays (35), (36) and (38) by a pair of light neutralinos. The latter would also remain unobservable and we would obtain similar signatures, possibly slightly modified by the light neutralino mass. We thus have potential new decay modes which can be used to search for, or constrain, the light neutralino scenario [3, 95, 96, 108-114, 203, 209]. The mass range of the decaying mesons (e.g. $m_{\pi} \approx 130 \mathrm{MeV}, m_{K} \approx 500 \mathrm{MeV}$, $m_{\Upsilon} \approx 10 \mathrm{GeV}$ ) enables a test of various light neutralino masses [95].

Motivated by our discussion in Sects. 2 and 4, we assume throughout this section that the neutralino is pure bino, denoted as $\widetilde{B}$, and very light, i.e. that the neutralino decay mode is kinematically accessible to the meson. We recall that a pure bino has no interaction with the $Z$ boson and its couplings to right-handed sfermions are stronger than to left-handed sfermions. The corresponding supersymmetric Feynman diagrams for the meson decays can be quite different from those for the SM decays. We list a few examples in Fig. 11.

In the following, we give an overview over the present bounds existing in the literature, modifying them to binos, where necessary. The analyses carried out so far involve significant approximations. An up-to-date, detailed analysis of meson decays to light neutralinos, in particular including the necessary higher-order calculations, is beyond the scope of this paper and will be presented in [116]. However, given the current status of the data, cf. Table 2, we expect the present conclusions to be robust.

\subsection{Pseudoscalar mesons}

Pseudoscalar mesons (psm), e.g. $\pi^{0}, K, \eta, \eta^{\prime}$, and $B_{s}$ can in principle decay into a light neutralino pair. The related photino decay $\pi^{0} \rightarrow \tilde{\gamma} \tilde{\gamma}$ was first computed in [3, 96]. Later, in [108, 109], the decays $\pi^{0} \rightarrow \tilde{\gamma} \tilde{\gamma}$ and $\pi^{0} \rightarrow \tilde{\gamma} \tilde{\gamma} \gamma$ 
were investigated. If we rescale the result in [108, 109] from a squark mass of $m_{\tilde{q}}=70 \mathrm{GeV}$ to a squark mass of $m_{\tilde{q}}=300 \mathrm{GeV}$ [212], we obtain an upper bound on the photino branching ratios, which is well below the current experimental limits for the neutrino decays [40]:

$$
\begin{aligned}
& \operatorname{BR}\left(\pi^{0} \rightarrow \nu \bar{v}\right) \lesssim 2.7 \times 10^{-7}, \\
& \operatorname{BR}\left(\pi^{0} \rightarrow \nu \bar{v} \gamma\right) \lesssim 6 \times 10^{-4} .
\end{aligned}
$$

This conclusion also holds for the corresponding bino decays. For their theoretical estimates the authors in [108, 109] assumed a large left-right mixing in the squark sector, not taking into account flavour changing neutral current constraints. Due to the very small supersymmetric branching ratio, no bound is obtained on the bino mass.

In $[3,96]$ no left-right mixing was assumed. We reestimate their branching ratio for a pure bino and for heavier squarks and assume that the relevant squarks are degenerate. We also generalise to an arbitrary psm. The corresponding Feynman graph for a pion is shown in Fig. 11(a). The decay width to a bino pair is then given by

$$
\begin{aligned}
\Gamma(\mathrm{psm} \rightarrow \tilde{B} \tilde{B})= & \frac{\pi \alpha^{2}}{4 \cos ^{4} \theta_{\mathrm{w}}} C(\mathrm{psm}) \frac{m_{\widetilde{B}}^{2} m_{\mathrm{psm}}}{m_{\tilde{q}}^{4}} \\
& \times \sqrt{1-\frac{4 m_{\widetilde{B}}^{2}}{m_{\mathrm{psm}}^{2}}} .
\end{aligned}
$$

The constant $C$ depends on the considered meson and is given by

$$
\begin{aligned}
& C\left(\pi^{0}\right)=\frac{2}{9} f_{\pi^{0}}^{2}, \\
& C(\eta)=\frac{3}{4}\left(\sin \theta_{1} f_{1}-\frac{2 \sqrt{2}}{9} \cos \theta_{8} f_{8}\right)^{2}, \\
& C\left(\eta^{\prime}\right)=\frac{3}{4}\left(\sin \theta_{1} f_{1}+\frac{2 \sqrt{2}}{9} \cos \theta_{8} f_{8}\right)^{2}, \\
& C\left(B_{S}\right)=\frac{\left|V_{c b}\right|^{2} c^{2} f_{B_{s}}^{2}}{216 \pi^{2}},
\end{aligned}
$$

where $f_{\pi^{0}}, f_{1}, f_{8}$, and are the decay constant of the $\pi^{0}, \eta_{1}$, $\eta_{8}$, and $B_{s}$, respectively; $\theta_{1,8}$ are the mixing angles between the $\eta_{1}$ and $\eta_{8}$ states. $V_{c b}$ is the charm-bottom CKM matrix element. These are all given in [40,213]. $c$ parameterises the flavour mixing in the squark sector; representative values for $c$ and $f_{B_{s}}$ are taken from [110].

At the parton level, the decay $B_{s} \rightarrow \widetilde{B} \widetilde{B}$ proceeds via $b \rightarrow s \widetilde{B} \widetilde{B}$, cf. Fig. 11(b). The flavour changing neutral current (FCNC) is possible, because the left-handed squark mass matrices and the quark mass matrices are not (necessarily) simultaneously diagonal [110]. This mismatch is parameterised by the constant $c$. The authors of [110] obtain for a $80 \mathrm{GeV}$ squark mass a branching ratio of about $\mathcal{O}\left(10^{-7}-10^{-5}\right)$, depending on the amount of flavour violation. In Table 2, we have rescaled their result and present the branching ratio for a squark mass of $300 \mathrm{GeV}$.

In Table 2, we list in the upper part the numerical values for the branching ratios for the various meson decays to binos. Here we have assumed $m_{\tilde{q}}=300 \mathrm{GeV}$. Note that the decay width, (41), peaks at a value $m_{\widetilde{B}}=m_{\mathrm{psm}} / \sqrt{6}$. In order to obtain conservative bounds in Table 2 , we assume in turn for each meson this value for the bino mass. It can be seen that the branching ratios for a meson decay into a light bino pair are several orders of magnitude lower than the experimental bounds on the respective invisible widths. This is mainly due to the small $\widetilde{B}$ mass in the numerator (because of the helicity flip required for a decay of a pseudoscalar into a pair of binos) and the large squark masses in the denominator of (41), suppressing this weak decay mode. Consequently no bounds on a light $\widetilde{B}$ can be set from these decay modes.

\subsection{Vector mesons and quarkonium decay}

The decays of vector mesons to photinos were first considered in $[3,203,215]$. The decay of the various $\Upsilon(n S)$ (excited) states into a neutralino pair and its discovery possibility at $B$ factories is discussed in detail in $[113,114]$.

In [215], the decay of quarkonium into gluinos and photinos has been calculated. Theses decays violate parity. The coupling strength of photinos and gluinos to left- and righthanded squarks are equal. Consequently, the masses of lefthanded and right-handed squarks must be different to allow for these decays. However, the first argument does not hold for the decay into binos: the coupling strength to righthanded particles is larger than to left-handed particles, i.e. parity is explicitly violated. Hence, this decay is also possible when left- and right-handed squark masses are equal to each other.

The quarkonium decay width can be approximated by, cf. [218],

$$
\begin{aligned}
& \Gamma\left({ }^{3} S_{1}(q \bar{q}) \rightarrow \tilde{B} \tilde{B}\right) \\
& \quad=\frac{C_{V}}{\cos ^{4} \theta_{\mathrm{w}}} \Gamma\left(V \rightarrow e^{+} e^{-}\right) \frac{m_{V}^{4}}{m_{\tilde{q}}^{4}}\left(1-\frac{4 m_{\widetilde{B}}^{2}}{M_{V}^{2}}\right)^{3 / 2},
\end{aligned}
$$

where $m_{V}$ is the mass of the vector meson $V={ }^{3} S_{1}(q \bar{q})$, and $m_{\tilde{q}}$ is the mass of the exchanged squark. $C_{V}$ is a constant depending on the sum of the squared quark-squark-bino coupling strengths. For the different mesons, $C_{V}$ is given by

$$
\begin{aligned}
& C_{\rho}=\frac{289}{10368}, \quad m_{\tilde{u}_{L}}=m_{\tilde{d}_{R}} \rightarrow \infty, \\
& m_{\tilde{u}_{R}}=m_{\tilde{d}_{L}} \equiv m_{\tilde{q}}=300 \mathrm{GeV}
\end{aligned}
$$


Fig. 12 Examples for loop-induced supersymmetric kaon decays

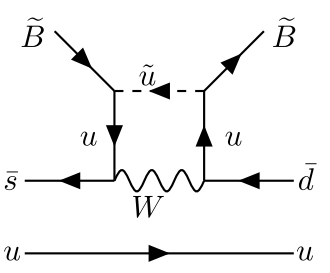

(a) Box diagram

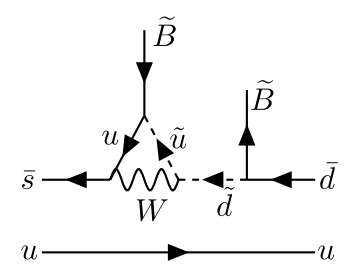

(b) Triangle diagram

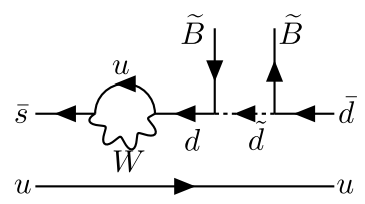

(c) Bubble Diagram
$C_{\omega}=\frac{25}{72}, \quad m_{\tilde{u}_{L}}=m_{\tilde{d}_{L}} \rightarrow \infty$,

$m_{\tilde{u}_{R}}=m_{\tilde{d}_{R}} \equiv m_{\tilde{q}}=300 \mathrm{GeV}$

$C_{\phi}=\frac{1}{72}, \quad m_{\tilde{s}_{L}} \rightarrow \infty$,

$m_{\tilde{s}_{R}} \equiv m_{\tilde{q}}=300 \mathrm{GeV}$

$C_{J / \psi}=\frac{1}{18}, \quad m_{\tilde{c}_{2}} \rightarrow \infty$,

$m_{\tilde{c}_{1}} \equiv m_{\tilde{q}}=300 \mathrm{GeV}, \quad \theta_{\tilde{c}}=\frac{\pi}{2}$

$C_{\Upsilon}=\frac{1}{72}, \quad m_{\tilde{b}_{2}} \rightarrow \infty$,

$m_{\tilde{c}_{1}} \equiv m_{\tilde{q}}=300 \mathrm{GeV}, \quad \theta_{\tilde{b}}=\frac{\pi}{2}$,

where we indicate the approximations we have made for the squark masses. In (47)-(51), we have also listed the input parameters (squark masses and mixing angles) employed to derive the upper bounds on the branching ratio. They are chosen in such a way that the branching ratio is maximal for a massless neutralino. For the $u, d$, and $s$ squark, we assume that left- and right-handed squarks do not mix and that all squark masses are degenerate. For the $c$ and $b$ squark, we include squark mixing. $\theta_{\tilde{c}}$ and $\theta_{\tilde{b}}$ denote the mixing angles in the scalar charm and bottom sector, respectively.

As in the pseudoscalar meson case, the decays are strongly suppressed by the large squark masses. The results of the theory evaluation are compared to the experimental data in the middle part of Table 2. It can be seen that no bounds on the mass of a light bino can be deduced. The discrepancy between the experimental bound and the theoretical estimate is quite large. We thus expect these results to be quite robust. More detailed formulæ can be found in [116].

\subsection{Loop-induced meson decays to binos}

In [95-97, 209], the related photino decay $K^{+} \rightarrow \pi^{+} \tilde{\gamma} \tilde{\gamma}$ was analysed. It can proceed at tree level via the cascade decay

$$
\begin{aligned}
K^{+} \rightarrow & \pi^{+} \pi^{0} \\
& \hookrightarrow \tilde{\gamma} \tilde{\gamma}
\end{aligned}
$$

It can also proceed directly at the loop level, for which example Feynman graphs are shown in Figs. 12(a)-(c). It was found that for large parts of the MSSM parameter space this decay is suppressed relative to the SM decay $K^{+} \rightarrow \pi^{+} \nu \bar{\nu}$. Since the SM event rate is barely observable with present experiments, no bound on the photino mass or the relevant sfermion masses in the propagators is obtained. However, these results were derived when the correct mass of the top quark was unknown. Furthermore, only an incomplete set of the relevant one-loop Feynman graphs was evaluated, and the neutralino was restricted to be a photino. Nonetheless, the estimate obtained using the published results is well below the experimental bound, and we do not expect any bound from present data for a bino. However, in [116] this issue will be investigated based on a complete analysis.

A similar analysis can be carried out for the decay $B^{+} \rightarrow$ $\pi \widetilde{B} \widetilde{B}$. The corresponding SUSY box diagrams involve $W^{ \pm}$ bosons, squarks, charginos, and Higgs bosons. As evaluated in $[95,96]$, the suppression by the scalar fermions in the loops (which are assumed to exceed the experimental bounds of $\sim 300 \mathrm{GeV}$ ) leads to a contribution that is too small to set any limits on a light bino.

Another rare decay in which a light bino could in principle play a role is $b \rightarrow s \gamma$. However, at the one-loop level neutralinos do not contribute. The effect of a light $\widetilde{B}$ at the two-loop level would be well within the current theoretical and experimental uncertainties [219].

\section{Astrophysical and cosmological bounds on the neutralino mass}

In this section we briefly consider the implications of a very light neutralino for astrophysics and cosmology. We focus on supernova cooling and the dark matter of the universe. In [220] the implications for a moderately light neutralino with $m_{\tilde{\chi}_{1}^{0}} \gtrsim 5 \mathrm{GeV}$ for Big Bang nucleosynthesis are discussed, which we shall not further consider here.

\subsection{Supernova cooling}

During a supernova explosion neutrinos are abundantly produced in the dense core. They diffuse out with a time 
scale of $\mathcal{O}(10 \mathrm{sec})$ [221-223]. The neutrinos with this time structure have indeed been observed after Supernova 1987a [224, 225]. If light neutralinos exist with mass less than or of order the supernova core temperature, $T_{c}=\mathcal{O}(30 \mathrm{MeV})$, they can also be produced abundantly during core collapse. Depending on their interactions, these neutralinos escape freely from the supernova, rapidly cooling the core [226]. As the temperature drops, the neutrino scattering cross section drops with the square of the temperature, leading eventually to free-streaming neutrinos. Thus rapid cooling of the supernova with a time scale well below $10 \mathrm{sec}$ is excluded by the neutrino observation from Supernova 1987a. This can be used to set restrictions on the light neutralino mass, as well as its interactions. This was originally addressed for photinos in [77-79]. The authors of [80] derived important lower bounds on a light bino, which we briefly summarise here; see also [81]. Our focus here is on a massless neutralino.

The two main neutralino production mechanisms in a supernova are electron-positron annihilation and nucleonnucleon $(N N)$ "neutralino-strahlung":

$e^{+}+e^{-} \longrightarrow \tilde{\chi}_{1}^{0}+\tilde{\chi}_{1}^{0}$,

$N+N \longrightarrow N+N+\tilde{\chi}_{1}^{0}+\tilde{\chi}_{1}^{0}$.

Once produced, the neutralinos have a mean-free-path, $\lambda_{\tilde{\chi}_{1}^{0}}$, in the supernova core which is determined via the cross sections for the processes

$\tilde{\chi}_{1}^{0}+e \longrightarrow \tilde{\chi}_{1}^{0}+e$,

$\tilde{\chi}_{1}^{0}+N \longrightarrow \tilde{\chi}_{1}^{0}+N$,

as well as the electron and nucleon densities. If $\lambda_{\tilde{\chi}_{1}^{0}}$ is of order of the core size, $R_{c}=\mathcal{O}(10 \mathrm{~km})$, or larger, the neutralinos escape freely and thus cool the supernova rapidly. However, if the neutralinos have masses $m_{\tilde{\chi}_{1}^{0}}$ much greater than the supernova core temperature $T_{c}$, then their production is Boltzmann-factor suppressed and they affect the cooling negligibly, independent of $\lambda_{\tilde{\chi}_{1}^{0}}$. Demanding that $m_{\tilde{\chi}_{1}^{0}}$ be large enough that neutralino cooling does not markedly alter the neutrino signal-particularly its time-structure-allows one to set a lower limit on the neutralino mass. Note that this limit depends strongly on the squark and selectron masses, which enter to the fourth power in (53), (54) and (55), (56) through the relevant propagators.

A proper treatment of this problem would be to expand the existing supernova code(s) to include the production and the scattering of neutralinos. Thus the neutralinos would be involved in the complete time evolution of the supernova, which could affect the particle densities within the supernova and the supernova temperature as a function of time. Such a treatment is beyond the scope of this paper. A good estimate of the effect of the neutralinos on the supernova evolution can be obtained, if we use the existing codes
[227-230] and treat this non-supersymmetric supernova as a fixed background, i.e. we assume the neutralino effect on the evolution to be small. Using the resulting electron and nucleon densities, we can compute the production and scattering of the light neutralinos. We then employ the Raffelt criterion [231], requiring that the maximal emitted energy from the supernova via neutralino radiation is $\leq 10^{52} \mathrm{erg}$. In [80], it was then found that for selectron masses in the range $300 \mathrm{GeV} \lesssim m_{\tilde{e}} \lesssim 900 \mathrm{GeV}$ neutralino masses below $100 \mathrm{MeV}$ are excluded. As the selectron mass is increased from $900 \mathrm{GeV}$ the lower bound an the neutralino mass gradually decreases. For selectron masses above $1.2 \mathrm{TeV}$ there is no lower bound on the lightest neutralino mass.

Similar, however much less restrictive, arguments also hold for the squark mass dependence. For a massless neutralino, squark masses between $300 \mathrm{GeV}$ and $360 \mathrm{GeV}$ are excluded.

For selectron and squark masses below $300 \mathrm{GeV}$ the mean-free-path of the neutralino is smaller than the supernova core size: $\lambda_{\tilde{\chi}_{1}^{0}}<R_{c}$, i.e. the neutralinos are trapped, and diffuse out, just like the neutrinos. In this case the above approximate procedure is no longer valid and the neutralinos must be included in the numerical supernova simulation. This has to-date not been performed. Thus, at present, massless neutralinos are not excluded by the Supernova 1987a observations for $m_{\tilde{e}}, m_{\tilde{q}}<300 \mathrm{GeV}$ or both $m_{\tilde{e}}>1200$ $\mathrm{GeV}$ and $m_{\tilde{q}}>360 \mathrm{GeV}$.

It should be pointed out that to-date it is not yet possible to successfully simulate a full supernova explosion; in particular the outgoing shock wave still stalls. Thus some ingredient is still missing. More recently the simulations are being extended to three dimensions with the inclusion of turbulent effects [232, 233]. An eventual full solution could in principle lead to a modification of the above results.

\subsection{Dark matter}

In the MSSM with conserved proton hexality or conserved $R$-parity, the lightest neutralino is stable and will contribute to the dark matter in the universe [21, 234-237]. If it is very light, i.e. relativistic at freeze-out, it will contribute hot dark matter. If the dark matter candidate is non-relativistic at freeze-out, it contributes to the cold dark matter of the universe. The structure formation in the early universe is best described by cold dark matter alone [238]. Thus the contribution of hot dark matter to the energy density of the universe is severely restricted. We discuss the resulting bound on the light neutralino mass, the Cowsik-McClelland bound, in Sect. 6.2.1.

For cold dark matter, it is well known that for smaller interaction cross sections the resulting relic density is larger. Furthermore the reaction rate also decreases with decreasing mass of the dark matter candidate. Therefore, if we assume 
that the candidate particle provides the required cold dark matter of the universe, we obtain a lower bound on the particle mass, the Lee-Weinberg bound. We shall discuss the resulting bound for a neutralino in Sect. 6.2.2. This bound assumes the standard big-bang cosmology with a radiation dominated universe prior to nucleosynthesis. If one drops this assumption, it has recently been shown that the lower bound is substantially weakened [54]. If for example one generalises the MSSM to the next-to-minimal supersymmetric Standard Model (NMSSM), which contains an extra singlet chiral superfield, the bounds can also be weakened [64, $65,113,114]$. We restrict ourselves to the MSSM and the standard big-bang cosmology.

\subsubsection{The Cowsik-McClelland bound}

Here, we consider the case of a (nearly) massless neutralino, $m_{\tilde{\chi}_{1}^{0}} \lesssim \mathcal{O}(1 \mathrm{eV})$. As for meson decays above, we restrict ourselves to a pure bino neutralino [89, 90]. Since the very light bino contributes to the hot dark matter of the universe, we assume here implicitly that the cold dark matter originates from another source; see e.g. [76] for a review of alternate candidates. For a sufficiently light bino the contribution of the hot dark matter to the energy density of the universe is expected to be consistent with present observations. We wish here to quantify this statement and thus determine an upper mass bound on a very light stable bino.

Note that if we were to assume that proton hexality or $R$-parity is violated, then for such a light bino $\left[m_{\tilde{\chi}_{1}^{0}} \lesssim\right.$ $\mathcal{O}(1 \mathrm{eV})]$, kinematically the only open decay mode is $\widetilde{B} \rightarrow v \gamma$ (provided a lighter neutrino exists), which proceeds via a one-loop diagram. Using the computation in [239], we can estimate the lifetime in this case as

$\tau_{\widetilde{B}} \approx \frac{1}{\lambda^{2}} 10^{-9} \sec \left(\frac{m_{\tilde{f}}}{100 \mathrm{GeV}}\right)^{4}\left(\frac{1 \mathrm{GeV}}{m_{\widetilde{B}}}\right)^{3}$,

where $\lambda$ is the relevant $R$-parity violating coupling [33-35], and $m_{\tilde{f}}$ is the mass of the sfermion entering the loop. For $\lambda=0.01$ (a typical upper bound), $m_{\tilde{f}}=100 \mathrm{GeV}$ and a bino mass $m_{\widetilde{B}}=1 \mathrm{eV}$, we obtain a lifetime of about $10^{22} \mathrm{sec}$, well above the age of the universe.

For a stable bino, the bino relic energy density, $\rho_{\widetilde{B}}$, divided by the critical energy density of the universe, $\rho_{c}$, is given by [240]

$\Omega_{\widetilde{B}} \equiv \frac{\rho_{\widetilde{B}}}{\rho_{c}}=\frac{43}{11} \frac{\zeta(3)}{\pi^{2}} \frac{8 \pi G_{N}}{3 H_{0}^{2}} \frac{g_{\text {eff }}(\widetilde{B})}{g_{* S}(T)} T_{\gamma}^{3} m_{\widetilde{B}}$.

$G_{N}$ and $H_{0}$ denote Newton's gravitation constant and the present value of the Hubble constant, respectively. $\zeta(3)$ is the Riemann zeta function evaluated at 3. $T_{\gamma}$ is the photon temperature. Recall the effective internal degrees of freedom of a particle are given in terms of the internal degrees of freedom, $g$, by

$g_{\text {eff }}= \begin{cases}g, & \text { for a boson, } \\ \frac{3}{4} g, & \text { for a fermion. }\end{cases}$

For the bino, which is a Majorana fermion, we have $g_{\text {eff }}(\widetilde{B})=2 \times(3 / 4)=1.5 \cdot g_{* S}$ is given by

$g_{* S}=\sum_{i=\text { bosons }} g_{i} \cdot\left(\frac{T_{i}}{T}\right)^{3}+\frac{7}{8} \sum_{i=\text { fermions }} g_{i} \cdot\left(\frac{T_{i}}{T}\right)^{3}$.

Here, $g_{i}$ is the number of internal degrees of freedom and $T_{i}$ the temperature of the particle species $i$, respectively. The sum runs over the index $i$ for all species in thermal equilibrium at temperature $T$.

In order for the bino hot dark matter not to disturb the structure formation, we conservatively assume its contribution to the total energy density of the universe to be less than the upper bound on the energy density of the neutrinos, as determined by the WMAP data $[74,75]$,

$\Omega_{\widetilde{B}} h^{2} \leq\left[\Omega_{v} h^{2}\right]_{\max }=0.0076$.

Light binos decouple at $T \approx \mathcal{O}(1-10 \mathrm{MeV})$. This temperature is somewhat higher than the temperature where the neutrinos decouple, because the selectron mass is larger than $M_{W}$. The higher selectron mass leads to a smaller bino scattering cross section, i.e. earlier freeze-out. However, the temperature is well below the muon mass, so it is not necessary to know the exact value. Nevertheless, at this temperature, we have two bosonic and 12 fermionic relativistic degrees of freedom (one photon, one Dirac electron, three left-handed neutrino species, and one light Majorana neutralino) leading to $g_{* S}=12.5$. From (58) and (61), we find the conservative upper bound

$m_{\widetilde{B}} \leq 0.7 \mathrm{eV}$.

Thus a very light bino with mass below about $1 \mathrm{eV}$ is consistent with structure formation. This line of argument was originally used by Gershtein and Zel'dovich [241] and Cowsik and McClelland [242] to derive a neutrino upper mass bound, by requiring $\Omega_{v} \leq 1$. We have here obtained an upper mass bound for a hot dark matter bino.

\subsubsection{The Lee-Weinberg bound}

In this subsection, we determine the lower mass bound on a light neutralino from the requirement that it alone provides the required cold dark matter in the universe. This is based on the original work for massive neutrinos [243-246] and the resulting bound is referred to as the Lee-Weinberg bound in the literature. As a light neutralino, we consider 
Fig. 13 (Color online) Constant contours of the relic density of a bino type LSP as a function of the LSP mass, $m_{\widetilde{B}}$, and the slepton mass, $m_{\tilde{\ell}}$. In the red (dark) shaded area the relic density is in the allowed range $0.091<\Omega_{D M} h^{2}<0.129$.

The grey (light) shaded areas are excluded due to LEP searches, $m_{\tilde{\ell}}<80 \mathrm{GeV}$ and the theoretical requirement: $m_{\tilde{\ell}}<m_{\widetilde{B}}$

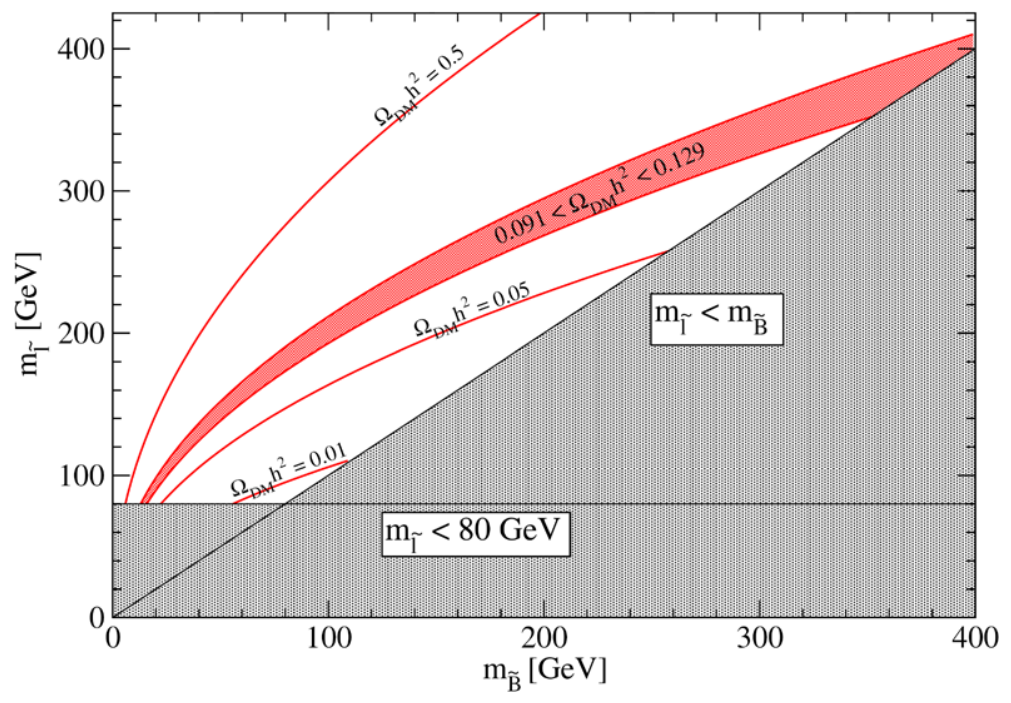

here a neutralino with a mass below the LEP bound, (1), but which is nevertheless non-relativistic in the early universe, i.e. neutralinos with $m_{\tilde{\chi}_{1}^{0}} \geq \mathcal{O}(5 \mathrm{GeV})$. For simplicity, we shall again restrict ourselves to the case of a pure bino, as we are mainly interested in qualitative statements. This neutralino mass range has been investigated numerically in $[45-50,53]$ in some detail. We present here a semianalytical treatment of the Boltzmann equation following [240]. This gives some insight into the dependence of the neutralino relic density on the main parameters in our case: the bino mass $m_{\widetilde{B}}$, and the slepton mass $m_{\tilde{\ell}}$. At the end of this section, we compare our results to [45-49,53].

For simplicity, we shall consider only the neutralino annihilation into leptons,

$\widetilde{B} \widetilde{B} \rightarrow \ell \bar{\ell}, \quad \ell=e, \mu, \tau, v_{e}, v_{\mu}, v_{\tau}$.

The $\tau$-lepton is considered as massless, all sleptons are assumed to have common mass $m_{\tilde{\ell}}$. The relevant annihilation cross sections are then related by

$$
\begin{aligned}
\sigma\left(\widetilde{B} \widetilde{B} \rightarrow \ell_{R}^{-} \bar{\ell}_{L}^{+}\right) & =16 \sigma\left(\widetilde{B} \widetilde{B} \rightarrow \ell_{L}^{-} \bar{\ell}_{R}^{+}\right) \\
& =16 \sigma\left(\widetilde{B} \widetilde{B} \rightarrow v_{\ell} \bar{\nu}_{\ell}\right) .
\end{aligned}
$$

The cross section averaged over the thermal distributions of the incoming particles is given by [240,247]

$$
\begin{aligned}
\langle\sigma(\widetilde{B} \widetilde{B} & \rightarrow \ell \bar{\ell})|v|\rangle \approx \sigma_{0} x^{-1} \equiv 54 \pi \frac{\alpha^{2}}{c_{w}^{4}} \frac{m_{\widetilde{B}}^{2}}{m_{\tilde{\ell}}^{4}} x^{-1}, \\
\text { with } x & \equiv \frac{m_{\widetilde{B}}}{T} .
\end{aligned}
$$

Here $T$ is the temperature of the universe. Implementing our specific cross section into the procedure outlined in Chap. 5.2 of [240], we obtain for the bino freeze-out tem- perature, $T_{f}$,

$$
\begin{aligned}
x_{f} \equiv & \frac{m_{\widetilde{B}}}{T_{f}} \approx \ln \left[\frac{0.19 \cdot g}{g_{*}^{1 / 2}}\left(\frac{x\langle\sigma|v|\rangle s}{H\left(m_{\widetilde{B}}\right)}\right)_{x=1}\right] \\
& -\frac{3}{2} \ln \left[\ln \left(\frac{0.19 \cdot g}{g_{*}^{1 / 2}}\left(\frac{x\langle\sigma|v|\rangle s}{H\left(m_{\widetilde{B}}\right)}\right)_{x=1}\right)\right] .
\end{aligned}
$$

Here the thermally averaged cross section is evaluated at $x=$ 1, i.e. $T=m_{\widetilde{B}}$. The effective number of relativistic degrees of freedom $g_{*}$ is given by

$g_{*}=\sum_{i=\text { bosons }} g_{i}\left(\frac{T_{i}}{T}\right)^{4}+\frac{7}{8} \sum_{i=\text { fermions }} g_{i}\left(\frac{T_{i}}{T}\right)^{4}$.

$H\left(m_{\widetilde{B}}\right)$ is the Hubble parameter for $T=m_{\widetilde{B}}$. The bino contribution to the normalised energy density of the universe is then given by

$\Omega_{\widetilde{B}} h^{2}=m_{\widetilde{B}} n_{\widetilde{B}} \approx \frac{2.14 \times 10^{9} x_{f}^{2}}{\left(g_{* S} / g_{*}^{1 / 2}\right) m_{\mathrm{Pl}} \sigma_{0}} \mathrm{GeV}^{-1}$.

Here $m_{\mathrm{Pl}}$ denotes the Planck mass. We have plotted in Fig. 13 contours of constant relic density in the $m_{\widetilde{B}}-m_{\tilde{\ell}}$ plane. The lower right-hand triangle of the figure is excluded since here the sleptons are lighter than the neutralino, contrary to our assumption. The bottom horizontal band, $m_{\tilde{\ell}}<80 \mathrm{GeV}$, is excluded due to LEP searches [40]. The red (dark shaded) band denotes the preferred relic density region from the 5-year WMAP data [75]

$\Omega_{\widetilde{B}} h^{2}=0.1099 \pm 3 \sigma_{\Omega}=0.1099 \pm 0.0186$,

where we have implemented the 3 sigma bounds, and where the 1 sigma error is $\sigma_{\Omega}=0.0062$. 
As expected, we see in (68) that the resulting relic density is inversely proportional to the bino annihilation cross section. Furthermore

$\Omega_{\widetilde{B}} h^{2} \propto \frac{m_{\tilde{\ell}}^{4}}{m_{\widetilde{B}}^{2}}$.

Thus for fixed $\Omega_{\widetilde{B}} h^{2}$ the slepton mass $m_{\tilde{\ell}} \propto \sqrt{m_{\widetilde{B}}}$. This can be seen in Fig. 13. Since observationally there exists an upper bound $\Omega_{\widetilde{B}} h^{2}<\left[\Omega_{\widetilde{B}} h^{2}\right]_{\max }=0.129$, this translates into a lower bound on the bino mass $m_{\widetilde{B}}$. We obtain the most conservative bound for the smallest allowed slepton mass $m_{\tilde{\ell}}=80 \mathrm{GeV}$. Inserting these numbers we obtain

$m_{\widetilde{B}}>13 \mathrm{GeV}$.

From Fig. 13 it should also be clear that one obtains an upper bound on $m_{\widetilde{B}}$ for $m_{\tilde{\ell}}=m_{\widetilde{B}}$ and $\Omega_{\widetilde{B}} h^{2}=\left[\Omega_{\widetilde{B}} h^{2}\right]_{\max }$,

$m_{\widetilde{B}}<419 \mathrm{GeV}$.

This is then also the upper bound on the slepton mass in this scenario.

The neutralino relic density has been considered extensively in the literature; see for example [21-23, 234-237]. More recently the question has been raised of precisely how light the lightest neutralino can be, while still providing the entire (cold) dark matter in the universe as required by the WMAP data [45-50]. The authors allowed for non-universal gaugino masses, cf. (2), as well as a general neutralino admixture. They took into account all kinematically allowed annihilation products, as well as co-annihilation and resonant annihilation. In [45] a lower bound of about $18 \mathrm{GeV}$ was found for a non-relativistic neutralino. The lowest lower bound of about $6 \mathrm{GeV}$ was found in $[48,49]$ in models with a light pseudoscalar Higgs $A$ with mass $M_{A}<200 \mathrm{GeV}$. In $[47,49]$ a semi-analytical approximation was also performed. Here one focused on the $s$-channel Higgs exchange specifically in the low $M_{A}$ region. It is encouraging how well these numbers agree with our approximate results, which only considered the neutralino annihilation to leptons. This is mainly because the resonant and co-annihilation effects we have ignored are not relevant in the low-mass region.

In a further study, it was found that when allowing for explicit CP violation in the Higgs, and thus the neutralino sector the lower bound is reduced to $3 \mathrm{GeV}$ [53].

\section{Summary and conclusion}

In this paper we have studied mass bounds on light neutralinos from collider physics, precision observables, meson decays, and astrophysics and cosmology. We have focused on the question how light the $\tilde{\chi}_{1}^{0}$ can be, in particular whether an essentially massless $\tilde{\chi}_{1}^{0}$ is allowed by the experimental and observational data. Assuming a semi-simple gauged Lie group and that the parameters $M_{1}$ and $M_{2}$ are independent. A very light neutralino is possible by mildly tuning $M_{1}$, resulting in a substantial bino component of the $\tilde{\chi}_{1}^{0}$.

Specifically, we have analysed the processes $e^{+} e^{-} \rightarrow$ $\tilde{\chi}_{1}^{0} \tilde{\chi}_{2}^{0}$ and $e^{+} e^{-} \rightarrow \tilde{\chi}_{1}^{0} \tilde{\chi}_{1}^{0} \gamma$ for a massless neutralino. The LEP searches have determined an upper bound on the cross section of the first process. This reaction depends on the selectron masses, due to the $t$-channel selectron exchange contribution. Consequently, we can translate the upper bound on the $\tilde{\chi}_{1}^{0} \tilde{\chi}_{2}^{0}$ production cross section into a lower mass bound on the selectron masses, depending on the choice of $\mu$ and $M_{2}$. Assuming degenerate selectron masses and making the conservative assumption that the branching ratio of $\tilde{\chi}_{2}^{0}$ into $\tilde{\chi}_{1}^{0}$ and a $Z$ boson is $100 \%$, for $\mu<150 \mathrm{GeV}$ we find $m_{\tilde{e}} \gtrsim 1 \mathrm{TeV}$. For $\mu \gtrsim 200 \mathrm{GeV}$ no bounds on $m_{\tilde{e}}$ are obtained. As a consequence, this search channel yields no bounds on the mass of the lightest neutralino. Radiative neutralino production, $e^{+} e^{-} \rightarrow \tilde{\chi}_{1}^{0} \tilde{\chi}_{1}^{0} \gamma$, which has been searched for at LEP 2 and $b$-factories, also does not result in any bound on $m_{\tilde{\chi}_{1}^{0}}$.

Electroweak precision observables such as the $W$ boson mass, $M_{W}$, the effective leptonic weak mixing angle, $\sin ^{2} \theta_{\text {eff }}$, or the invisible $Z$ width, $\Gamma_{\text {inv }}$ are potentially sensitive to a very light neutralino. For $M_{W}$ and $\sin ^{2} \theta_{\text {eff }}$, where the neutralino contribution is a loop effect, we find that even the most precise anticipated measurements will not be able to constrain the mass of $\tilde{\chi}_{1}^{0}$. The contribution to the invisible $Z$ width, arising from the decay of the $Z$ boson into a pair of very light neutralinos, can give rise to a measurable effect if the bino component of the $Z$ boson is not too high. Also this constraint, however, is not very powerful for most of the parameter space, and the largest effects occur in parameter regions that are already excluded by the LEP searches for charginos. We have furthermore analysed the effect of a light neutralino on electric dipole moments and the anomalous magnetic moment of the muon. Both do not show any relevant sensitivity to $m_{\tilde{\chi}_{1}^{0}}$.

Next we investigated the bounds from rare meson decays. Pseudo-scalar mesons and vector mesons can decay into a pair of massless neutralinos. This decay, however, is suppressed by (large) squark masses and, in the case of pseudoscalar mesons, a small neutralino mass. This decay has the same signature as the SM decay into a neutrino pair. Using the corresponding experimental upper bounds no constraints on $m_{\tilde{\chi}_{1}^{0}}$ could be established. We also found no bound on $m_{\tilde{\chi}_{1}^{0}}$ from the rare, loop-induced cascade decay $K^{+} \rightarrow \pi^{+} \tilde{\chi}_{1}^{0} \tilde{\chi}_{1}^{0}$.

We next considered the astrophysical bounds on the neutralino mass from neutralino production in a supernova explosion. We found that a massless neutralino is consistent with the neutrino observations of supernova 1987a, provided 
the selectron mass (which enters in the production and scattering cross sections) is larger than $1.2 \mathrm{TeV}$ and the squark mass is larger than $360 \mathrm{GeV}$. However, for squark and selectron masses below $300 \mathrm{GeV}$ the neutralinos are trapped in the supernova, similar to the neutrinos. This scenario has not yet been sufficiently analyzed, so that also in this case a massless neutralino cannot be ruled out at present.

Finally, we considered cosmological bounds on the neutralino mass. If the neutralino constitutes hot dark matter it is in agreement with the WMAP observations and structure formation for a mass below $1 \mathrm{eV}$. For the case that the lightest neutralino constitutes the entire cold dark matter of the universe, we performed a semi-analytical analysis and found a lower bound of $m_{\tilde{\chi}_{1}^{0}}>13 \mathrm{GeV}$. This is in remarkably good agreement with the more complete numerical computations which find a lower bound of about $6 \mathrm{GeV}$.

Overall, we have found that a massless neutralino is consistent with all present laboratory experiments and astrophysical and cosmological observations. At the upcoming experiments at the LHC very light neutralinos could be produced in cascade decays of heavier supersymmetric particles. The anticipated precision in the determination of the differences between squared masses could allow one to further test scenarios with very light neutralinos. Further insights can be expected from high-precision measurements at a future $e^{+} e^{-}$Linear Collider.

Acknowledgements We would like to thank Sebastian Grab, Daniel Koschade, Michael Krämer, Greg Landsberg, Ben O'Leary, Peter Richardson and Subir Sarkar for numerous discussions on various aspects of this paper. HD would like to thank the Aspen centre for Physics where some of this work was performed. HD and UL would like to thank Christoph Hanhart and Daniel Phillips for the earlier collaboration on light neutralino supernova bounds. The work of HD, OK and UL was supported by SFB TR-33 The Dark Universe. The work of SH was partially supported by CICYT (grant FPA2006-02315). Work supported in part by the European Community's Marie-Curie Research Training Network under contract MRTN-CT-2006-035505 'Tools and Precision Calculations for Physics Discoveries at Colliders' (HEPTOOLS). This work has been supported by MICINN project FPA.2006-05294.

\section{References}

1. http://lhc.web.cern.ch/lhc/

2. J. Ellis, AIP Conf. Proc. 957, 38 (2007). arXiv:0710.0777 [hep-ph]

3. H. Haber, G. Kane, Phys. Rep. 117, 75 (1985)

4. H. Nilles, Phys. Rep. 110, 1 (1984)

5. M. Drees, R. Godbole, P. Roy, Theory and Phenomenology of Sparticles (World Scientific, Hackensack, 2004)

6. H. Baer, X. Tata, Weak Scale Supersymmetry: From Superfields to Scattering Events (Cambridge University Press, Cambridge, 2006).

7. S. Raby, Phys. Lett. B 422, 158 (1998). arXiv:hep-ph/9712254

8. H. Baer, K. Cheung, J. Gunion, Phys. Rev. D 59, 075002 (1999). arXiv:hep-ph/9806361
9. A. Akeroyd, M. Diaz, J. Ferrandis, M. Garcia-Jareno, J. Valle, Nucl. Phys. B 529, 3 (1998). arXiv:hep-ph/9707395

10. M. Hirsch, W. Porod, Phys. Rev. D 68, 115007 (2003). arXiv:hep-ph/0307364

11. A. de Gouvea, A. Friedland, H. Murayama, Phys. Rev. D 59, 095008 (1999). arXiv:hep-ph/9803481

12. C. Chou, H. Lai, C. Yuan, Phys. Lett. B 489, 163 (2000). arXiv:hep-ph/0006313

13. A. de Gouvea, S. Gopalakrishna, W. Porod, J. High Energy Phys. 0611, 050 (2006). arXiv:hep-ph/0606296

14. H. Dreiner, C. Luhn, M. Thormeier, Phys. Rev. D 73, 075007 (2006). arXiv:hep-ph/0512163

15. H. Dreiner, C. Luhn, H. Murayama, M. Thormeier, Nucl. Phys. B 795, 172 (2008). arXiv:0708.0989 [hep-ph]

16. G. Farrar, P. Fayet, Phys. Lett. B 76, 575 (1978)

17. R. Barate et al. (ALEPH Collaboration), Z. Phys. C 76, 1 (1997)

18. J. Abdallah et al. (DELPHI Collaboration), Eur. Phys. J. C 26, 505 (2003). arXiv:hep-ex/0303024

19. P. Janot, Phys. Lett. B 564, 183 (2003). arXiv:hep-ph/0302076

20. T. Hebbeker, Phys. Lett. B 470, 259 (1999). arXiv:hep-ph/ 9910326

21. J. Ellis, J. Hagelin, D. Nanopoulos, K. Olive, M. Srednicki, Nucl. Phys. B 238, 453 (1984)

22. H. Pagels, J. Primack, Phys. Rev. Lett. 48, 223 (1982)

23. H. Goldberg, Phys. Rev. Lett. 50, 1419 (1983)

24. See for example: J. Ellis, K. Olive, Y. Santoso, V. Spanos, Phys. Lett. B 588, 7 (2004). arXiv:hep-ph/0312262

25. J. Feng, S. Su, F. Takayama, Phys. Rev. D 70, 075019 (2004). arXiv:hep-ph/0404231

26. F. Steffen, J. Cosmol. Astropart. Phys. 0609, 001 (2006). arXiv:hep-ph/0605306

27. See for example: K. Rajagopal, M. Turner, F. Wilczek, Nucl. Phys. B 358, 447 (1991)

28. E. Chun, J. Kim, H. Nilles, Phys. Lett. B 287, 123 (1992). arXiv:hep-ph/9205229

29. L. Covi, J. Kim, L. Roszkowski, Phys. Rev. Lett. 82, 4180 (1999). arXiv:hep-ph/9905212

30. L. Ibanez, G. Ross, Nucl. Phys. B 368, 3 (1992)

31. H. Dreiner, C. Luhn, H. Murayama, M. Thormeier, Nucl. Phys. B 774, 127 (2007). arXiv:hep-ph/0610026

32. H. Dreiner, J. Soo Kim, M. Thormeier, arXiv:0711.4315 [hep-ph]

33. B. Allanach, A. Dedes, H. Dreiner, Phys. Rev. D 69, 115002 (2004). Erratum-ibid. D 72 (2005) 079902. arXiv:hep-ph/ 0309196

34. B. Allanach, M. Bernhardt, H. Dreiner, C. Kom, P. Richardson, Phys. Rev. D 75, 035002 (2007). arXiv:hep-ph/0609263

35. F. de Campos et al., arXiv:0712.2156 [hep-ph]

36. H. Dreiner, S. Grab, arXiv:0811.0200 [hep-ph]

37. E. Chun, H. Kim, Phys. Rev. D 60, 095006 (1999). arXiv:hep-ph/ 9906392

38. H. Lee, K. Matchev, T. Wang, Phys. Rev. D 77, 015016 (2008). arXiv:0709.0763 [hep-ph]

39. H. Lee, C. Luhn, K. Matchev, J. High Energy Phys. 0807, 065 (2008). arXiv:0712.3505 [hep-ph]

40. C. Amsler et al. (Particle Data Group), Phys. Lett. B 667, 1 (2008)

41. J. Abdallah et al. (DELPHI Collaboration), Eur. Phys. J. C 31, 421 (2004). arXiv:hep-ex/0311019

42. B. Greene, K. Kirklin, P. Miron, G. Ross, Nucl. Phys. B 278, 667 (1986)

43. L. Ibanez, J. Kim, H. Nilles, F. Quevedo, Phys. Lett. B 191, 282 (1987)

44. H. Dreiner, J. Lopez, D. Nanopoulos, D. Reiss, Nucl. Phys. B 320, 401 (1989)

45. D. Hooper, T. Plehn, Phys. Lett. B 562, 18 (2003). arXiv: hep-ph/0212226 
46. G. Belanger, F. Boudjema, A. Pukhov, S. Rosier-Lees, arXiv: hep-ph/0212227

47. A. Bottino, N. Fornengo, S. Scopel, Phys. Rev. D 67, 063519 (2003). arXiv:hep-ph/0212379

48. G. Belanger, F. Boudjema, A. Cottrant, A. Pukhov, S. RosierLees, J. High Energy Phys. 0403, 012 (2004). arXiv:hep-ph/ 0310037

49. A. Bottino, F. Donato, N. Fornengo, S. Scopel, Phys. Rev. D 68 , 043506 (2003). arXiv:hep-ph/0304080

50. A. Bottino, F. Donato, N. Fornengo, S. Scopel, Phys. Rev. D 69, 037302 (2004). arXiv:hep-ph/0307303

51. A. Bottino, F. Donato, N. Fornengo, S. Scopel, Phys. Rev. D 70, 015005 (2004). arXiv:hep-ph/0401186

52. A. Bottino, F. Donato, N. Fornengo, S. Scopel, Phys. Rev. D 77, 015002 (2008). arXiv:0710.0553 [hep-ph]

53. J. Lee, S. Scopel, Phys. Rev. D 75, 075001 (2007). arXiv:hep-ph/ 0701221

54. S. Profumo, Phys. Rev. D 78, 023507 (2008). arXiv:0806.2150 [hep-ph]

55. A. Salam, J. Strathdee, Nucl. Phys. B 87, 85 (1975)

56. P. Fayet, Nucl. Phys. B 90, 104 (1975)

57. A. Chamseddine, H. Dreiner, Nucl. Phys. B 458, 65 (1996). arXiv:hep-ph/9504337

58. D. Castano, D. Freedman, C. Manuel, Nucl. Phys. B 461, 50 (1996). arXiv:hep-ph/9507397

59. D. Freedman, Phys. Rev. D 15, 1173 (1977)

60. R. Barbieri, L. Maiani, Nucl. Phys. B 243, 429 (1984)

61. R. Barbieri, L. Girardello, A. Masiero, Phys. Lett. B 127, 429 (1983).

62. L. Hall, L. Randall, Nucl. Phys. B 352, 289 (1991)

63. E. Dudas, S. Lavignac, J. Parmentier, Nucl. Phys, Nucl. Phys. B 808, 237 (2009). arXiv:0808.0562 [hep-ph]

64. V. Barger, P. Langacker, H. Lee, Phys. Lett. B 630, 85 (2005). arXiv:hep-ph/0508027

65. J. Gunion, D. Hooper, B. McElrath, Phys. Rev. D 73, 015011 (2006). arXiv:hep-ph/0509024

66. D. Chung, G. Farrar, E. Kolb, Phys. Rev. D 56, 6096 (1997). arXiv:astro-ph/9703145

67. E. Kolb, A. Riotto, Phys. Rev. D 54, 3722 (1996). arXiv:astro-ph/ 9601096

68. G. Farrar, E. Kolb, Nucl. Phys. Proc. Suppl. B 51, 188 (1996)

69. S. White, C. Frenk, M. Davis, Astrophys. J. 274, L1 (1983)

70. G. Smoot et al. Astrophys. J. 396, L1 (1992)

71. D. Spergel et al. (WMAP Collaboration), Astrophys. J. Suppl. 148, 175 (2003). arXiv:astro-ph/0302209

72. K. Abazajian, Phys. Rev. D 73, 063513 (2006). arXiv:astro-ph/ 0512631

73. M. Viel, G. Becker, J. Bolton, M. Haehnelt, M. Rauch, W. Sargent, Phys. Rev. Lett. 100, 041304 (2008). arXiv:0709.0131 [astro-ph]

74. D. Spergel et al. (WMAP Collaboration), Astrophys. J. Suppl. 170, 377 (2007). arXiv:astro-ph/0603449

75. J. Dunkley et al. (WMAP Collaboration), arXiv:0803.0586 [astro-ph]

76. F. Steffen, arXiv:0811.3347 [hep-ph]

77. J. Grifols, E. Masso, S. Peris, Phys. Lett. B 220, 591 (1989)

78. J. Ellis, K. Olive, S. Sarkar, D. Sciama, Phys. Lett. B 215, 404 (1988)

79. K. Lau, Phys. Rev. D 47, 1087 (1993)

80. H. Dreiner, C. Hanhart, U. Langenfeld, D. Phillips, Phys. Rev. D 68, 055004 (2003). arXiv:hep-ph/0304289

81. M. Kachelriess, J. High Energy Phys. 0002, 010 (2000). arXiv:hep-ph/0001160

82. D. Sciama, Phys. Lett. B 112, 211 (1982)

83. D. Sciama, Phys. Lett. B 114, 19 (1982)

84. D. Sciama, Phys. Lett. B 121, 119 (1983)
85. P. Fayet, Phys. Lett. B 117, 460 (1982)

86. J. Ellis, J. Hagelin, Phys. Lett. B 122, 303 (1983)

87. H. Dreiner, O. Kittel, U. Langenfeld, Phys. Rev. D 74, 115010 (2006). arXiv:hep-ph/0610020

88. H. Dreiner, O. Kittel, U. Langenfeld, Eur. Phys. J. C 54, 277 (2008). arXiv:hep-ph/0703009

89. D. Choudhury, H. Dreiner, P. Richardson, S. Sarkar, Phys. Rev. D 61, 095009 (2000). arXiv:hep-ph/9911365

90. A. Dedes, H. Dreiner, P. Richardson, Phys. Rev. D 65, 015001 (2002). arXiv:hep-ph/0106199

91. P. Chankowski, A. Dabelstein, W. Hollik, W. Mösle, S. Pokorski, J. Rosiek, Nucl. Phys. B 417, 101 (1994)

92. W. de Boer, A. Dabelstein, W. Hollik, W. Mösle, U. Schwickerath, Z. Phys. C 75, 627 (1997). arXiv:hep-ph/9607286

93. J. Erler, D. Pierce, Nucl. Phys. B 526, 53 (1998). arXiv:hep-ph/ 9801238

94. S. Heinemeyer, W. Hollik, G. Weiglein, Phys. Rep. 425, 265 (2006). arXiv:hep-ph/0412214

95. J. Ellis, J. Hagelin, Nucl. Phys. B 217, 189 (1983)

96. M. Gaillard, Y. Kao, I. Lee, M. Suzuki, Phys. Lett. B 123, 241 (1983)

97. J. Nieves, P. Pal, Phys. Rev. D 32, 1849 (1985)

98. S. Adler et al. (E787 Collaboration), Phys. Rev. Lett. 79, 2204 (1997). arXiv:hep-ex/9708031

99. S. Adler et al. (E787 Collaboration), Phys. Rev. Lett. 88, 041803 (2002). arXiv:hep-ex/0111091

100. V. Anisimovsky et al. (E949 Collaboration), Phys. Rev. Lett. 93, 031801 (2004). arXiv:hep-ex/0403036

101. S. Adler et al. (E787 Collaboration), Phys. Rev. D 77, 052003 (2008)

102. A.J. Buras, M. Gorbahn, U. Haisch, U. Nierste, Phys. Rev. Lett. 95, 261805 (2005). arXiv:hep-ph/0508165

103. A.J. Buras, M. Gorbahn, U. Haisch, U. Nierste, J. High Energy Phys. 0611, 002 (2006). arXiv:hep-ph/0603079

104. G. Isidori, F. Mescia, C. Smith, Nucl. Phys. B, 718, 319 (2005). arXiv:hep-ph/0503107

105. F. Mescia, C. Smith, Phys. Rev. D 76, 034017 (2007). arXiv: 0705.2025 [hep-ph]

106. J. Brod, M. Gorbahn, Phys. Rev. D 78, 034006 (2008). arXiv:0805.4119[hep-ph]

107. P. Fayet, Nucl. Phys. B 78, 14 (1974)

108. M. Dobroliubov, A. Ignatiev, V. Matveev, Sov. J. Nucl. Phys. 47, 296 (1988); [Yad. Fiz. 47, 468 (1988)]

109. M. Dobroliubov, A. Ignatiev, V. Matveev, Phys. Lett. B 192, 135 (1987). Note: These are the same papers

110. R. Adhikari, B. Mukhopadhyaya, Phys. Rev. D 52, 3125 (1995). arXiv:hep-ph/9411347

111. R. Adhikari, B. Mukhopadhyaya, Phys. Lett. B 353, 228 (1995). arXiv:hep-ph/9411208

112. R. Adhikari, B. Mukhopadhyaya, arXiv:hep-ph/9508256

113. B. McElrath, Phys. Rev. D 72, 103508 (2005). arXiv:hep-ph/ 0506151

114. B. McElrath, in The Proceedings of International Workshop on Charm Physics (Charm 2007), Ithaca, New York, 5-8 Aug. 2007, pp. 19. arXiv:0712.0016 [hep-ph]

115. L. Chang, O. Lebedev, J. Ng, Phys. Lett. B 441, 419 (1998). arXiv:hep-ph/9806487

116. H. Dreiner, S. Grab, M. Krämer, U. Langenfeld, B. O’Leary, arXiv:0905.2051 [hep-ph]

117. H. Dreiner, H. Haber, S. Martin, arXiv:0812.1594 [hep-ph]

118. A. Bartl, H. Fraas, W. Majerotto, N. Oshimo, Phys. Rev. D 40, 1594 (1989)

119. I. Gogoladze, J. Lykken, C. Macesanu, S. Nandi, Phys. Rev. D 68, 073004 (2003). arXiv:hep-ph/0211391

120. H. Dreiner, S. Heinemeyer, O. Kittel, U. Langenfeld, A. Weber, G. Weiglein, arXiv:0707.1425 [hep-ph] 
121. T. Fritzsche, W. Hollik, Eur. Phys. J. C 24, 619 (2002). arXiv:hep-ph/0203159

122. W. Öller, H. Eberl, W. Majerotto, C. Weber, Eur. Phys. J. C 29, 563 (2003). arXiv:hep-ph/0304006

123. T. Adams et al. (CDF Collaboration and D0 Collaboration), arXiv:0808.0728 [hep-ex]

124. A. Bottino, N. Fornengo, G. Polesello, S. Scopel, Phys. Rev. D 77, 115026 (2008). arXiv:0801.3334 [hep-ph]

125. G. Abbiendi et al. (OPAL Collaboration), Eur. Phys. J. C 35, 1 (2004). arXiv:hep-ex/0401026

126. S. Olsen, private communication

127. G. Moortgat-Pick et al. Phys. Rep. 460, 131 (2008). arXiv: hep-ph/0507011

128. S. Heinemeyer, W. Hollik, A. Weber, G. Weiglein, J. High Energy Phys. 0804, 039 (2008). arXiv:0710.2972 [hep-ph]

129. The ALEPH, DELPHI, L3, OPAL, SLD Collaborations, the LEP Electroweak Working Group, the SLD Electroweak and Heavy Flavour Groups, arXiv:hep-ex/0509008

130. The ALEPH, DELPHI, L3 and OPAL Collaborations, the LEP Electroweak Working Group, arXiv:hep-ex/0511027; see also: http://lepewwg.web.cern.ch/LEPEWWG/Welcome.html

131. S. Heinemeyer, W. Hollik, D. Stöckinger, A. Weber, G. Weiglein, J. High Energy Phys. 08, 052 (2006). arXiv:hep-ph/0604147

132. A. Djouadi, P. Gambino, S. Heinemeyer, W. Hollik, C. Jünger, G. Weiglein, Phys. Rev. Lett. 78, 3626 (1997). arXiv:hep-ph/ 9612363

133. A. Djouadi, P. Gambino, S. Heinemeyer, W. Hollik, C. Jünger, G. Weiglein, Phys. Rev. D 57, 4179 (1998). arXiv:hep-ph/ 9710438

134. S. Heinemeyer, G. Weiglein, J. High Energy Phys. 0210, 072 (2002). arXiv:hep-ph/0209305

135. S. Heinemeyer, G. Weiglein, arXiv:hep-ph/0301062

136. J. Haestier, S. Heinemeyer, D. Stöckinger, G. Weiglein, J. High Energy Phys. 0512, 027 (2005). arXiv:hep-ph/0508139

137. J. Haestier, S. Heinemeyer, D. Stöckinger, G. Weiglein, hep-ph/ 0506259

138. M. Awramik, M. Czakon, A. Freitas, G. Weiglein, Phys. Rev. D 69, 053006 (2004). arXiv:hep-ph/0311148

139. M. Awramik, M. Czakon, A. Freitas, J. High Energy Phys. 11, 048 (2006). arXiv:hep-ph/0608099

140. A. Freitas, W. Hollik, W. Walter, G. Weiglein, Phys. Lett. B 495, 338 (2000). Erratum-ibid. B 570, 260 (2003). arXiv:hep-ph/ 0007091

141. A. Freitas, W. Hollik, W. Walter, G. Weiglein, Nucl. Phys.B 632, 189 (2002). Erratum-ibid. B 666, 305 (2003). arXiv:hep-ph/ 0202131

142. M. Awramik, M. Czakon, Phys. Lett. B 568, 48 (2003). arXiv: hep-ph/0305248

143. M. Awramik, M. Czakon, Phys. Rev. Lett. 89, 241801 (2002). arXiv:hep-ph/0208113

144. M. Awramik, M. Czakon, Nucl. Phys. Proc. Suppl. 116, 238 (2003). arXiv:hep-ph/0211041

145. A. Onishchenko, O. Veretin, Phys. Lett. B 551, 111 (2003). arXiv:hep-ph/0209010

146. M. Awramik, M. Czakon, A. Onishchenko, O. Veretin, Phys. Rev. D 68, 053004 (2003). arXiv:hep-ph/0209084

147. W. Hollik, U. Meier, S. Uccirati, Nucl. Phys. B 731, 213 (2005). arXiv:hep-ph/0507158

148. M. Awramik, M. Czakon, A. Freitas, G. Weiglein, Phys. Rev. Lett. 93, 201805 (2004). arXiv:hep-ph/0407317

149. M. Awramik, M. Czakon, A. Freitas, G. Weiglein, Nucl. Phys. Proc. Suppl. 135, 119 (2004). arXiv:hep-ph/0408207

150. M. Awramik, M. Czakon, A. Freitas, G. Weiglein, arXiv:hep-ph/ 0409142

151. W. Hollik, U. Meier, S. Uccirati, Phys. Lett. B 632, 680 (2006). arXiv:hep-ph/0509302
152. W. Hollik, U. Meier, S. Uccirati, Nucl. Phys.B 765, 154 (2007). arXiv:hep-ph/0610312

153. M. Czakon, M. Awramik, A. Freitas, Nucl. Phys. Proc. Suppl. 157, 58 (2006). arXiv:hep-ph/0602029

154. M. Czakon, M. Awramik, A. Freitas, Phys. Lett. B 642, 563 (2006). arXiv:hep-ph/0605339

155. A. Djouadi, C. Verzegnassi, Phys. Lett. B 195, 265 (1987)

156. A. Djouadi, Nuovo Cimento A 100, 357 (1988)

157. L. Avdeev et al. Phys. Lett. B 336, 560 (1994). Erratum-ibid. B 349, 597 (1995). arXiv:hep-ph/9406363

158. K. Chetyrkin, J. Kühn, M. Steinhauser, Phys. Lett. B 351, 331 (1995). arXiv:hep-ph/9502291

159. J. van der Bij, K. Chetyrkin, M. Faisst, G. Jikia, T. Seidensticker, Phys. Lett. B 498, 156 (2001). arXiv:hep-ph/0011373

160. R. Boughezal, J. Tausk, J. van der Bij, Nucl. Phys. B 713, 278 (2005). arXiv:hep-ph/0410216

161. M. Faisst, J. Kühn, T. Seidensticker, O. Veretin, Nucl. Phys. B 665, 649 (2003). arXiv:hep-ph/0302275

162. Y. Schröder, M. Steinhauser, Phys. Lett. B 622, 124 (2005) arXiv:hep-ph/0504055

163. K. Chetyrkin, M. Faisst, J. Kühn, P. Maierhofer, C. Sturm, Phys. Rev. Lett. 97, 102003 (2006). arXiv:hep-ph/0605201

164. R. Boughezal, M. Czakon, Nucl. Phys. B 755, 221 (2006). arXiv:hep-ph/0606232

165. G. Wilson, LC-PHSM-2001-009, see: http://www.desy.de/ $\sim$ lcnotes/notes.html

166. U. Baur, R. Clare, J. Erler, S. Heinemeyer, D. Wackeroth, G. Weiglein, D. Wood, arXiv:hep-ph/0111314

167. R. Hawkings, K. Mönig, Eur. Phys. J. C 8, 1 (1999). arXiv: hep-ex/9910022

168. D. Chang, W. Keung, A. Pilaftsis, Phys. Rev. Lett. 82, 900 (1999). Erratum-ibid. 83, 3972 (1999). arXiv:hep-ph/9811202

169. A. Pilaftsis, Phys. Lett. B 471, 174 (1999). arXiv:hep-ph/ 9909485

170. O. Lebedev, K. Olive, M. Pospelov, A. Ritz, Phys. Rev. D 70, 016003 (2004). arXiv:hep-ph/0402023

171. P. Nath, Phys. Rev. Lett. 66, 2565 (1991)

172. Y. Kizukuri, N. Oshimo, Phys. Rev. D 46, 3025 (1992)

173. T. Ibrahim, P. Nath, Phys. Lett. B 418, 98 (1998). arXiv:hep-ph/ 9707409

174. T. Ibrahim, P. Nath, Phys. Rev. D 57, 478, (1998). Erratumibid. D 58, 019901 (1998). Erratum-ibid. D 60, 079903 (1998). Erratum-ibid. D 60, 119901 (1999). arXiv:hep-ph/9708456

175. M. Brhlik, G. Good, G. Kane, Phys. Rev. D 59, 115004 (1999). arXiv:hep-ph/9810457

176. S. Abel, S. Khalil, O. Lebedev, Nucl. Phys. B 606, 151 (2001). arXiv:hep-ph/0103320

177. D. Demir, O. Lebedev, K. Olive, M. Pospelov, A. Ritz, Nucl. Phys. B 680, 339 (2004). arXiv:hep-ph/0311314

178. T. Moroi, Phys. Rev. D 53, 6565 (1996). Erratum-ibid. D 56, 4424 (1997). arXiv:hep-ph/9512396

179. G. Degrassi, G. Giudice, Phys. Rev. D 58, 053007 (1998). arXiv:hep-ph/9803384

180. S. Heinemeyer, D. Stöckinger, G. Weiglein, Nucl. Phys. B 690, 62 (2004). arXiv:hep-ph/0312264

181. S. Heinemeyer, D. Stöckinger, G. Weiglein, Nucl. Phys. B 699, 103 (2004). arXiv:hep-ph/0405255

182. D. Stöckinger, J. Phys. G 34, R45 (2007). arXiv:hep-ph/0609168

183. S. Heinemeyer, W. Hollik, G. Weiglein, Comput. Phys. Commun. 124, 76 (2000). arXiv:hep-ph/9812320

184. The code is accessible via http://www.feynhiggs.de

185. S. Heinemeyer, W. Hollik, G. Weiglein, Eur. Phys. J. C 9, 343 (1999). arXiv:hep-ph/9812472

186. G. Degrassi, S. Heinemeyer, W. Hollik, P. Slavich, G. Weiglein, Eur. Phys. J. C 28, 133 (2003). arXiv:hep-ph/0212020 
187. M. Frank, T. Hahn, S. Heinemeyer, W. Hollik, H. Rzehak, G. Weiglein, J. High Energy Phys. 02, 047 (2007). arXiv:hep-ph/ 0611326

188. M. Davier, arXiv:hep-ph/0701163

189. F. Jegerlehner, Acta Phys. Pol. B 38, 3021 (2007). arXiv:hep-ph/ 0703125

190. K. Hagiwara, A. Martin, D. Nomura, T. Teubner, Phys. Lett. B 649, 173 (2007). arXiv:hep-ph/0611102

191. J. Miller, E. de Rafael, B. Roberts, Rep. Prog. Phys. 70, 795 (2007). arXiv:hep-ph/0703049

192. B. Regan, E. Commins, C. Schmidt, D. DeMille, Phys. Rev. Lett. 88, 071805 (2002)

193. C. Baker et al. Phys. Rev. Lett. 97, 131801 (2006). arXiv:hep-ex/ 0602020

194. M. Romalis, W. Griffith, E. Fortson, Phys. Rev. Lett. 86, 2505 (2001). arXiv:hep-ex/0012001

195. B. Kayser, G. Garvey, E. Fischbach, S. Rosen, Phys. Lett. B 52, 385 (1974)

196. P. Herczeg, C. Hoffman, Phys. Lett. B 100, 347 (1981). Erratumibid. B 102, 445 (1981)

197. J. Rich, D. Winn, Phys. Rev. D 14, 1283 (1976)

198. D. Besson et al. (CLEO Collaboration), Phys. Rev. D 30, 1433 (1984)

199. R. Balest et al. (CLEO Collaboration), Phys. Rev. D 51, 2053 (1995)

200. M. Ablikim et al. (BES Collaboration), Phys. Rev. Lett. 97, 202002 (2006). arXiv:hep-ex/0607006

201. M. Ablikim et al. (BES Collaboration), Phys. Rev. Lett. 100, 192001 (2008). arXiv:0710.0039 [hep-ex]

202. P. Fayet, Phys. Lett. B 84, 421 (1979)

203. B. Campbell, J. Scott, M. Sundaresan, Phys. Lett. B 131, 213 (1983)

204. P. Fayet, J. Kaplan, Phys. Lett. B 269, 213 (1991)

205. P. Fayet, Phys. Rev. D 74, 054034 (2006). arXiv:hep-ph/ 0607318

206. T. Inami, C. Lim, Prog. Theor. Phys. 65, 297 (1981). Erratumibid. 651772 (1981)

207. G. Buchalla, A. Buras, Nucl. Phys. B 400, 225 (1993)

208. S. Singh, L. Wolfenstein, Nucl. Phys. B 24, 77 (1970)

209. W. Keung, Phys. Rev. D 28, 1129 (1983)

210. A. Buras, A. Romanino, L. Silvestrini, Nucl. Phys. B 520, 3 (1998). arXiv:hep-ph/9712398

211. N. Deshpande, D. Ghosh, X. He, Phys. Rev. D 70, 093003 (2004). arXiv:hep-ph/0407021

212. V. Abazov et al. (D0 Collaboration), Phys. Lett. B 660, 449 (2008). arXiv:0712.3805 [hep-ex]

213. W. Yao et al. (Particle Data Group), J. Phys. G 33, 1 (2006)

214. A. Artamonov et al. (E949 Collaboration), Phys. Rev. D 72, 091102 (2005). arXiv:hep-ex/0506028

215. J. Ellis, S. Rudaz, Phys. Lett. B 128, 248 (1983)

216. O. Tajima et al. (Belle Collaboration), Phys. Rev. Lett. 98, 132001 (2007). arXiv:hep-ex/0611041
217. P. Rubin et al. (CLEO Collaboration), Phys. Rev. D 75, 031104 (2007). arXiv:hep-ex/0612051

218. V. Barger, R. Phillips, Collider Physics (Addison-Wesley, Redwood City, 1987), 592 p. (Frontiers in Physics, 71)

219. G. Isidori, private communication, 2008

220. K. Jedamzik, Phys. Rev. D 70, 083510 (2004). arXiv:astro-ph/ 0405583

221. D. Freedman, Phys. Rev. D 9, 1389 (1974)

222. T. Mazurek, Astrophys. J. 207, L87 (1976)

223. K. Sato, Prog. Theor. Phys. 54, 1325 (1975)

224. K. Hirata et al. (KAMIOKANDE-II Collaboration), Phys. Rev. Lett. 58, 1490 (1987)

225. R. Bionta et al. Phys. Rev. Lett. 58, 1494 (1987)

226. R. Gandhi, A. Burrows, Phys. Lett. B 246, 149 (1990). Erratumibid. B 261, 519 (1991)

227. A. Burrows, J. Lattimer, Astrophys. J. 307, 178 (1986)

228. J. Pons, S. Reddy, M. Prakash, J. Lattimer, J. Miralles, Astrophys. J. 513, 780 (1999). arXiv:astro-ph/9807040

229. A. Mezzacappa, M. Liebendoerfer, O. Messer, W. Hix, F. Thielemann, S. Bruenn, Phys. Rev. Lett. 86, 1935 (2001). arXiv: astro-ph/0005366

230. R. Buras, M. Rampp, H. Janka, K. Kifonidis, Astron. Astrophys. 447, 1049 (2006). arXiv:astro-ph/0507135

231. G. Raffelt, Stars as Laboratories for Fundamental Physics: The Astrophysics of Neutrinos, Axions, and Other Weakly Interacting Particles (University Press, Chicago, 1996), 664 p.

232. L. Scheck, T. Plewa, H. Janka, K. Kifonidis, E. Mueller, Phys. Rev. Lett. 92, 011103 (2004). arXiv:astro-ph/0307352

233. H. Janka, B. Mueller, F. Kitaura, R. Buras, arXiv:0712.4237 [astro-ph]

234. K. Griest, Phys. Rev. D 38, 2357 (1988). Erratum-ibid. D 39, 3802 (1989)

235. G. Jungman, M. Kamionkowski, K. Griest, Phys. Rep. 267, 195 (1996). arXiv:hep-ph/9506380

236. M. Drees, M.M. Nojiri, D.P. Roy, Y. Yamada, Phys. Rev. D 56, 276 (1997). Erratum-ibid. D 64, 039901 (2001). arXiv:hep-ph/ 9701219

237. J.R. Ellis, K.A. Olive, Y. Santoso, V.C. Spanos, Phys. Lett. B 565, 176 (2003). arXiv:hep-ph/0303043

238. G. Blumenthal, S. Faber, J. Primack, M. Rees, Nature 311, 517 (1984)

239. S. Dawson, Nucl. Phys. B 261, 297 (1985)

240. E. Kolb, M. Turner, The Early Universe (Westview Press, 1990)

241. S. Gershtein, Y. Zeldovich, JETP Lett. 4, 120 (1966); [Pisma Zh. Eksp. Teor. Fiz. 4, 174 (1966)]

242. R. Cowsik, J. McClelland, Phys. Rev. Lett. 29, 669 (1972)

243. B. Lee, S. Weinberg, Phys. Rev. Lett. 39, 165 (1977)

244. P. Hut, Phys. Lett. B 69, 85 (1977)

245. K. Sato, M. Kobayashi, Prog. Theor. Phys. 58, 1775 (1977)

246. M. Vysotsky, A. Dolgov, Y. Zeldovich, JETP Lett. 26, 188 (1977); [Pisma Zh. Eksp. Teor. Fiz. 26, 200 (1977)]

247. P. Gondolo, G. Gelmini, Nucl. Phys. B 360, 145 (1991). 